\title{
Constructing lattice-free gradient polyhedra in dimension two ${ }^{\star}$
}

\author{
Joseph Paat ${ }^{\dagger}$, Miriam Schlöter ${ }^{\dagger}$, \\ Emily Speakman *
}

\begin{abstract}
Lattice-free gradient polyhedra can be used to certify optimality for mixed integer convex minimization models. We consider how to construct these polyhedra for unconstrained models with two integer variables under the assumption that all level sets are bounded. A classic result of Bell, Doignon, and Scarf states that a lattice-free gradient polyhedron with at most four facets exists in this setting. We present an algorithm for creating a sequence of gradient polyhedra, each of which has at most four facets, that finitely converges to a lattice-free gradient polyhedron. Each update requires constantly many gradient evaluations. Our updates imitate the gradient descent algorithm, and consequently, it yields a gradient descent type of algorithm for problems with two integer variables.
\end{abstract}

\section{Introduction.}

Let $f: \mathbb{R}^{d} \times \mathbb{R}^{n} \rightarrow \mathbb{R}$ be convex and differentiable with gradient $\nabla f: \mathbb{R}^{d} \times \mathbb{R}^{n} \rightarrow$ $\mathbb{R}^{d} \times \mathbb{R}^{n}$. We assume oracle access to $\nabla f$. The unconstrained mixed integer convex minimization problem is

$$
\min \left\{f(x, z):(x, z) \in \mathbb{R}^{d} \times \mathbb{Z}^{n}\right\} .
$$

Applications of (CM) include statistical regression and the closest vector problem. In this paper we consider how to solve (CM) by constructing an optimality certificate in the form of a lattice-free gradient polyhedron. The gradient

\footnotetext{
* This paper extends work from an IPCO paper of the same title. This version includes complete proofs as well as new results on convergence for functions that are Lipschitz continuous and strongly convex.

† Department of Mathematics, ETH Zürich, Switzerland

E-mail: \{joseph.paat, miriam.schloeter\}@ifor.math.ethz.ch

* Department of Mathematical and Statistical Sciences, University of Colorado Denver, USA

E-mail: emily.speakman@ucdenver.edu
} 
polyhedron corresponding to a non-empty finite set $\mathcal{U} \subseteq \mathbb{R}^{d} \times \mathbb{Z}^{n}$ is

$$
\operatorname{GP}(\mathcal{U}):=\left\{(x, z) \in \mathbb{R}^{d} \times \mathbb{R}^{n}: \nabla f(\bar{x}, \bar{z})^{\top}(x-\bar{x}, z-\bar{z}) \leq 0 \forall(\bar{x}, \bar{z}) \in \mathcal{U}\right\} .
$$

We say that $\operatorname{GP}(\mathcal{U})$ is lattice-free if $\operatorname{intr}(\operatorname{GP}(\mathcal{U})) \cap \mathbb{R}^{d} \times \mathbb{Z}^{n}=\emptyset$, where

$$
\operatorname{intr}(\operatorname{GP}(\mathcal{U})):=\left\{(x, z) \in \mathbb{R}^{d} \times \mathbb{R}^{n}: \nabla f(\bar{x}, \bar{z})^{\top}(x-\bar{x}, z-\bar{z})<0 \forall(\bar{x}, \bar{z}) \in \mathcal{U}\right\} .
$$

This definition of interior implies that $\operatorname{intr}(\operatorname{GP}(\mathcal{U}))=\emptyset$ if there exists $(\bar{x}, \bar{z}) \in$ $\mathcal{U}$ such that $\nabla f(\bar{x}, \bar{z})=\mathbf{0}$. The significance of lattice-free gradient polyhedra comes from the definition of convexity:

$$
f(x, z) \geq f(\bar{x}, \bar{z})+\nabla f(\bar{x}, \bar{z})^{\top}(x-\bar{x}, z-\bar{z}) \quad \forall(x, z),(\bar{x}, \bar{z}) \in \mathbb{R}^{d} \times \mathbb{R}^{n} .
$$

If $\operatorname{GP}(\mathcal{U})$ is lattice-free, then $\mathcal{U}$ contains a minimizer of (CM).

Constructing lattice-free gradient polyhedra is well studied when $n=0$. In fact, the classic gradient descent algorithm is a search algorithm for a latticefree set in this setting. See [14, Chapter 9] for a standard discussion on gradient descent. Gradient descent updates a vector $x^{i} \in \mathbb{R}^{d}$ to $x^{i+1}=x^{i}-\alpha^{i} \nabla f\left(x^{i}\right)$, where $\alpha^{i}>0$. Under mild assumptions, the sequence $\left(x^{i}\right)_{i=0}^{\infty}$ converges to a vector $x^{*}$ with $\nabla f\left(x^{*}\right)=\mathbf{0}$. One can verify that

$$
\nabla f\left(x^{*}\right)=\mathbf{0} \text { if and only if } \operatorname{intr}\left(\operatorname{GP}\left(\left\{x^{*}\right\}\right)\right)=\emptyset,
$$

so $\left(x^{i}\right)_{i=0}^{\infty}$ corresponds to a sequence of gradient polyhedra $\left(\operatorname{GP}\left(\left\{x^{i}\right\}\right)\right)_{i=0}^{\infty}$ that converges to a lattice-free gradient polyhedron $\operatorname{GP}\left(\left\{x^{*}\right\}\right)$. Notably, each gradient polyhedron that gradient descent generates has at most $2^{n}=2^{0}=1$ many facets and the initial $x^{0}$ can be chosen arbitrarily. Also, the algorithm only requires gradient evaluations (not even function evaluations are required).

Lattice-free gradient polyhedra can also be constructed iteratively when $n=1$ and $d=0$. This certificate can be found by starting with an arbitrary $\mathcal{U}^{0}=\left\{z^{0}, z^{0}+1\right\} \subseteq \mathbb{Z}$ and updating it as follows:

$$
\mathcal{U}^{i+1}:= \begin{cases}\left\{z^{i}-1, z^{i}\right\} & \text { if } 0<\nabla f\left(z^{i}\right) \\ \left\{z^{i}+1, z^{i}+2\right\} & \text { if } \nabla f\left(z^{i}+1\right)<0 \\ \mathcal{U}^{i} & \text { if } \nabla f\left(z^{i}\right) \leq 0 \leq \nabla f\left(z^{i}+1\right) .\end{cases}
$$

The gradient comparisons ensure that $\mathcal{U}^{i}$ is updated by 'flipping' closer to the minimizer $x^{*}$ of the continuous relaxation of (CM). If $\mathcal{U}^{i+1}=\mathcal{U}^{i}$, then $\mathcal{U}^{i}=\left\{\left\lfloor x^{*}\right\rfloor,\left\lceil x^{*}\right\rceil\right\}$ and $\operatorname{GP}\left(\mathcal{U}^{i}\right)$ is lattice-free. This update procedure is not the most efficient way of obtaining $\left\{\left\lfloor x^{*}\right\rfloor,\left\lceil x^{*}\right\rceil\right\}$ as one can simply round $x^{*}$. However, the procedure does have the same properties as gradient descent: every gradient polyhedron has at most $2^{n}=2^{1}=2$ many facets, the initial set $\mathcal{U}^{0}$ is arbitrary, and each update only requires a constant number of gradient evaluations. Both the update and the rounding approach generalize to when $d \geq 1$, but they do not extend naturally if $n \geq 2$.

The known results on lattice-free gradient polyhedra when $n \geq 2$ are existential rather than algorithmic. It follows from the work of Bell, Doignon, 
and Scarf [12,17,23] that there exists $\mathcal{U} \subseteq \mathbb{Z}^{n}$ such that $|\mathcal{U}| \leq 2^{n}$ and $\operatorname{GP}(\mathcal{U})$ is lattice-free. Baes et al. [5] extend this to lattice-free gradient polyhedra for problems with additional convex constraints. Basu et al. 8 generalize gradient polyhedra further to so-called $S$-free sets.

The goal of this paper is to iteratively construct a lattice-free gradient polyhedron when $n \geq 2$. We aim to design an algorithm that serves as a mixed integer counterpart to the gradient descent in the following sense: it creates a sequence of gradient polyhedra that each have at most $2^{n}$ many facets and that converges to a lattice-free polyhedron, it only requires gradient evaluations, and the initial $\mathcal{U}$ can be arbitrarily chosen. We say such an algorithm is of gradient descent type.

We make the following assumption:

$\forall \alpha \in \mathbb{R}$, the level set $\left\{(x, z) \in \mathbb{R}^{d} \times \mathbb{R}^{n}: f(x, z) \leq \alpha\right\}$ is bounded.

Assumption (11) guarantees that a lattice-free optimality certificate exists and that (CM) has an optimal solution. This assumption captures families such as strictly convex and strongly convex functions. The techniques in this paper do not immediately extend to detect if this assumption is violated.

\subsection{Related work}

Lattice-free convex sets (not necessarily gradient polyhedra) have been heavily studied in the context of valid inequality generation for integer programs, see, e.g., 1, 6, 10, 11, 15, and the references therein. We also refer to [2,3,9, 16, 22, for the structure of inclusion-wise maximal lattice-free polyhedra. Algorithms for (CM) use techniques such as branch and bound (see, e.g., 20,21]), outer approximations (see, e.g., [13,18]), convex separation [19, Theorem 6.7.10], or improvement oracles 4 4. Each of these algorithms use non-gradient information or create polyhedral relaxations with potentially more than $2^{n}$ facets. Baes et al. (4) give a geometric algorithm for (CM) when $d=0$ and $n=2$ but explicitly use knowledge of a bounded set containing the minimum. In particular, they solve (CM) by subdividing a box $B$ containing a minimizer of (CM) and solving $O(\ln (B))$ many continuous problem.

\subsection{Statement of results}

Our main contribution is a gradient descent type algorithm when $d=0$ and $n=2$. Figure 1 provides an example. The algorithm creates a sequence of gradient polyhedra $\left(\operatorname{GP}\left(\mathcal{U}^{i}\right)\right)_{i=0}^{\infty}$ defined by unimodular sets. We say $\mathcal{U} \subseteq \mathbb{Z}^{2}$ is unimodular if

$$
\mathcal{U}:=\mathcal{U}(z, U):=\left\{z+U e: e \in\{0,1\}^{2}\right\},
$$

for $z \in \mathbb{Z}^{2}$ and a matrix $U \in \mathbb{Z}^{2 \times 2}$ with $|\operatorname{det}(U)|=1$. Table 1 outlines how we update $\mathcal{U}^{i}$ to $\mathcal{U}^{i+1}$. Every gradient polyhedron we generate has at most 

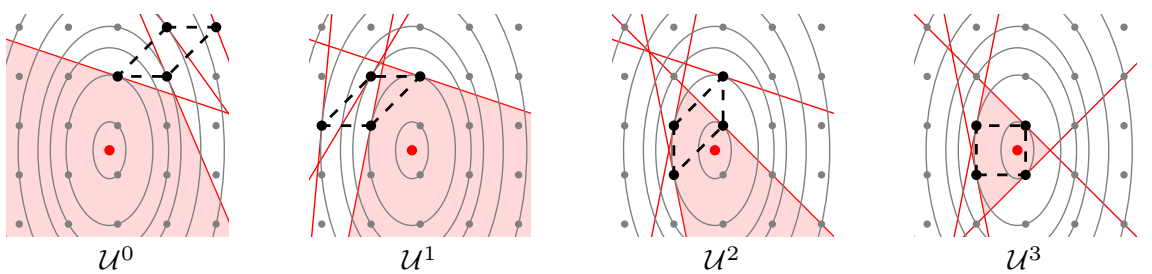

Fig. 1 A sequence $\left(\mathcal{U}^{i}\right)_{i=0}^{3}$ of unimodular sets generated by our updates for $f\left(x_{1}, x_{2}\right):=$ $3 x_{1}^{2}+x_{2}^{2}+x_{1}+x_{2}$. The convex hull of each $\mathcal{U}^{i}$ is outlined in black, and level curves of $f$ are in gray. The hyperplanes defining $\operatorname{GP}\left(\mathcal{U}^{i}\right)$ are in red, the minimizer of the continuous relaxation of (CM) is drawn as a red point, and $\operatorname{GP}\left(\mathcal{U}^{i}\right)$ is shaded in red.

$2^{n}=2^{2}=4$ many facets because it is defined by a unimodular set. Also, the initial set $\mathcal{U}^{0}$ can be any arbitrary unimodular set, and each update requires a constant number of gradient evaluations 1

We use two measures of progress to ensure that $\left(\mathcal{U}^{i}\right)_{i=0}^{\infty}$ converges to a lattice-free gradient polyhedron $\operatorname{GP}\left(\mathcal{U}^{T}\right)$. The first measure is the minimum function value in $\mathcal{U}^{i}$ :

$$
\min \left\{f(z): z \in \mathcal{U}^{i}\right\} .
$$

The second measure is the distance from the set of optimal solution of (CM) to $\mathcal{U}^{i}$ with respect to $U^{i}$ :

$$
\left.\min \left\{\left\|\left(U^{i}\right)^{-1}\left(z^{*}-z\right)\right\|_{1}: z \in \mathcal{U}^{i} \text { and } z^{*} \text { is optimal for (피 }\right)\right\} .
$$

We discuss (4) more in Section 3. Our updates are such that (3) and (4) are both non-increasing in $i$. Furthermore, if $\mathcal{U}^{i}$ does not contain a minimizer of (CM), then at least one measure strictly decreases after at most two updates. The unimodular set requires preprocessing, which is described in Lemma 2

Theorem 1 Let $\mathcal{U}^{i}$ be a preprocessed unimodular set. If $\mathcal{U}^{i}$ does not fit into Table 1, then it is lattice-free. Otherwise, it can be updated to a unimodular set $\mathcal{U}^{i+1}$ such that

(i) neither (3) nor (4) increases from $\mathcal{U}^{i}$ to $\mathcal{U}^{i+1}$,

(ii) if (3) and (4) remain constant and $\mathcal{U}^{i+1}$ is updated to $\mathcal{U}^{i+2}$, then

(ii-a) (3) or (41) strictly decreases from $\mathcal{U}^{i+1}$ to $\mathcal{U}^{i+2}$ or

(ii-b) $\mathcal{U}^{i}$ contains a minimizer of (CM).

Moreover, this can all be checked using constantly many gradient evaluations.

Assumption (1) implies that (3) and (4) can only strictly decrease a finite number of times. Therefore, if $\left(\mathcal{U}^{i}\right)_{i=0}^{\infty}$ is constructed using Table 1 , then $\operatorname{GP}\left(\mathcal{U}^{i}\right)$ is guaranteed to eventually contain a minimizer of (CM). The updates also guarantee that $\operatorname{GP}\left(\mathcal{U}^{i}\right)$ eventually becomes lattice-free.

1 We use 'gradient evaluation' to refer a single inner product evaluation using gradients, and 'constantly many' can be chosen to be 20 as counted in Table 1 
Theorem 2 Let $\mathcal{U}^{0}$ be unimodular and $\left(\mathcal{U}^{i}\right)_{i=0}^{\infty}$ be created using Table 1 . For some $T$ the gradient polyhedron $\operatorname{GP}\left(\mathcal{U}^{T}\right)$ is lattice-free, and this can be checked using constantly many gradient evaluations.

Our updates are conservative, but if $f$ is $L$-Lipschitz continuous and $c$ strongly convex, then only $(6 L / c+2) \cdot\left\|z^{*}\right\|_{1}$ many updates are needed to find an optimal solution $z^{*}$ (see Proposition 2). Furthermore, our updates can be extended to $d \geq 0$ provided we are able to exactly minimize $f_{z}(x):=f(x, z)$ over $x \in \mathbb{R}^{d}$ for each fixed $z \in \mathbb{Z}^{2}$. To see this, note that (CM) is the same as minimizing $f^{\min }(z):=\min \left\{f_{z}(x): x \in \mathbb{R}^{d}\right\}$ over $\mathbb{Z}^{2}$.

Corollary 1 The updates in Table 1 extend to a gradient descent type algorithm when $n=2$ and $d \geq 0$ if we can exactly minimize $f_{z}(x)$ over $\mathbb{R}^{d}$.

For the remainder of the paper, we assume $n=2$ and $d=0$. If a unimodular set $\mathcal{U}^{i}$ contains a vector $z^{*} \in \mathbb{Z}^{2}$ such that $\nabla f\left(z^{*}\right)=\mathbf{0}$, then $\operatorname{GP}\left(\mathcal{U}^{i}\right)$ is latticefree and $z^{*}$ is a minimizer of (CM). We always assume that this check is made and only consider updating $\mathcal{U}^{i}$ if the check fails.

\section{Preliminary results on convexity and gradient polyhedra corresponding to unimodular sets.}

We refer to [14 for more on convexity and gradients. Given $z, \bar{z} \in \mathbb{R}^{2}$, we say that $\bar{z}$ cuts $z$ if $\nabla f(\bar{z})^{\top}(z-\bar{z}) \geq 0$ and strictly cuts $z$ if $\nabla f(\bar{z})^{\top}(z-\bar{z})>0$. The next result follows from the definition of convexity.

Proposition 1 Let $z, \bar{z} \in \mathbb{R}^{2}$. If $\bar{z}$ cuts $z$ (respectively, strictly cuts), then $f(z) \geq f(\bar{z})$ (respectively, $f(z)>f(\bar{z})$ ).

We denote the $i$-th column of $U \in \mathbb{Z}^{2 \times 2}$ by $u^{i}$. The following result follows from Proposition 1 and the definition of gradient polyhedra.

Lemma 1 Let $\mathcal{X} \subseteq \mathbb{Z}^{2}$ be a non-empty finite set and $\mathcal{U}=\mathcal{U}(z, U)$ be unimodular. The following hold:

(i) If $z \notin \operatorname{intr}(\operatorname{GP}(\mathcal{X}))$, then $f(z) \geq \min \{f(\bar{z}): \bar{z} \in \mathcal{X}\}$.

(ii) $\mathcal{X} \cap \operatorname{GP}(\mathcal{X}) \neq \emptyset$.

(iii) If $\mathcal{U} \cap \operatorname{GP}(\mathcal{U})=\left\{z, z+u^{1}+u^{2}\right\}$, then $z+u^{i}$ does not strictly cut any vector in $\mathcal{U}$ for each $i \in\{1,2\}$.

(iv) If $z$ cuts $z+u^{1}$ and $z+u^{2}$, then $z$ strictly cuts $z+u^{1}+u^{2}$.

(v) If $z \in \operatorname{GP}(\mathcal{U})$ and no vector in $\mathcal{U}$ is strictly cut by $z$, then $|\mathcal{U} \cap \operatorname{GP}(\mathcal{U})| \geq 2$.

Let $\mathcal{U}=\mathcal{U}(z, U)$ be unimodular. After multiplying $u^{1}$ and $u^{2}$ by \pm 1 and relabeling the 'anchor' vector $z$ to be another vector in $\mathcal{U}$, we assume $\operatorname{GP}(\mathcal{U})$ fulfills preprocessing properties.

Lemma 2 Let $\mathcal{U}=\mathcal{U}(z, U)$ be unimodular. We can preprocess $\operatorname{GP}(\mathcal{U})$ so that (i) $z \in \operatorname{GP}(\mathcal{U})$, 
(ii) if $|\mathcal{U} \cap \mathrm{GP}(\mathcal{U})|=2$, then

(ii-a) $\mathcal{U} \cap \operatorname{GP}(\mathcal{U})=\left\{z, z+u^{1}\right\}$, or

(ii-b) $\mathcal{U} \cap \operatorname{GP}(\mathcal{U})=\left\{z, z+u^{1}+u^{2}\right\}$, z strictly cuts $z+u^{1}$, and $z+u^{1}+u^{2}$ strictly cuts $z+u^{2}$.

(iii) if $|\mathcal{U} \cap \mathrm{GP}(\mathcal{U})|=3$, then $\mathcal{U} \cap \operatorname{GP}(\mathcal{U})=\left\{z, z+u^{1}, z+u^{2}\right\}$.

Proof Lemma 1 (ii) states $\mathcal{U} \cap \operatorname{GP}(\mathcal{U}) \neq \emptyset$. Thus, we can relabel $\mathcal{U}$ so that $z \in \mathcal{U} \cap \mathrm{GP}(\mathcal{U})$ and $(i)$ holds. For (ii) and (iii), we refer to the following figure:

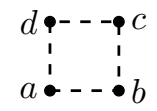

We have labeled the vectors in $\mathcal{U}$ as $a, b, c, d$. The vectors $b-a=c-d$ and $d-a=c-b$ are either $u^{1}$ or $u^{2}$ or a negative thereof. After possibly multiplying $u^{1}$ or $u^{2}$ by -1 , any three vectors in $\mathcal{U}$ can be relabeled to be $z, z+u^{1}$ and $z+u^{2}$. Thus, if $|\mathcal{U} \cap \operatorname{GP}(\mathcal{U})|=3$, then $\mathcal{U}$ can be relabeled to satisfy (iii) Similarly, if $\mathcal{U} \cap \operatorname{GP}(\mathcal{U})$ is equal to $\{a, b\},\{a, d\},\{b, c\}$, or $\{c, d\}$, then $\mathcal{U}$ can be relabeled so that $(i i-a)$ holds.

It is left to consider if $\mathcal{U} \cap \operatorname{GP}(\mathcal{U})$ is equal to $\{a, c\}$ or $\{b, d\}$. Without loss of generality suppose $\mathcal{U} \cap \operatorname{GP}(\mathcal{U})=\{a, c\}$. Relabel $a$ to be $z, c$ to be $z+u^{1}+u^{2}, b$ to be $z+u^{1}$ and $d$ to be $z+u^{2}$. Lemma 1 (iii) and (iv) imply that $z$ and $z+u^{1}+u^{2}$ each strictly cut exactly one of $z+u^{1}$ and $z+u^{2}$. After possibly relabeling one last time, we may assume that $z$ strictly cuts $z+u^{1}$ and $z+u^{1}+u^{2}$ strictly cuts $z+u^{2}$. This proves (ii-b)

We end the section with an observation.

Observation 1 Let $\mathcal{X} \subseteq \mathbb{Z}^{2}$ be a finite set. If $x_{1} \in \operatorname{intr}(\operatorname{GP}(\mathcal{X})), x_{2} \ldots, x_{t} \in$ $\operatorname{GP}(\mathcal{X}), \lambda_{1}, \ldots, \lambda_{t}>0$ and $\sum_{i=1}^{t} \lambda_{i}=1$, then $\sum_{i=1}^{t} \lambda_{i} x_{i} \in \operatorname{intr}(\operatorname{GP}(\mathcal{X}))$.

\section{Updating gradient polyhedra when $n=2$ and $d=0$.}

Let $\mathcal{U}=\mathcal{U}(z, U)$ be a unimodular set that satisfies the preprocessing of Lemma 2. We update $\mathcal{U}$ by replacing, or 'flipping', $U$ with a matrix $\bar{U}$ and then preprocessing $(z, \bar{U})$. We use $\operatorname{FLIP}(U)$ to denote the updated matrix $\bar{U}$, and $\operatorname{FLIP}(\mathcal{U})$ to denote the unimodular set obtained after preprocessing $(z, \bar{U})$. Table 1 defines FLIP $(U)$. Certain flips rely on the following line segments:

$$
H^{i}:=\left\{z+k u^{1}+i u^{2} \in \operatorname{intr}(\operatorname{GP}(\mathcal{U})): k \in \mathbb{R}\right\} \quad \forall i \in\{-1,1\} .
$$

We say that $\mathcal{U}$ is connected if $\mathcal{U} \cap \operatorname{GP}(\mathcal{U}) \supseteq\left\{z, z+u^{1}\right\}$ (Cases 3 to 5 ). Otherwise, we say $\mathcal{U}$ is disconnected (Cases 1 and 2). Note that $\mathcal{U}$ can be connected and not fit into Cases 3 to 5 this occurs when $\mathcal{U} \cap \operatorname{GP}(\mathcal{U})=\mathcal{U}$. On the other hand, if $\mathcal{U}$ is disconnected, then it must fit into the table. Note that if Case 1 is executed, then the values $\sigma^{1}, \sigma^{2}$ defined in the table cannot both equal one by Lemma $10(v)$.

The importance of the connected case is that we are able to quickly determine if $\operatorname{GP}(\mathcal{U})$ is lattice-free. 


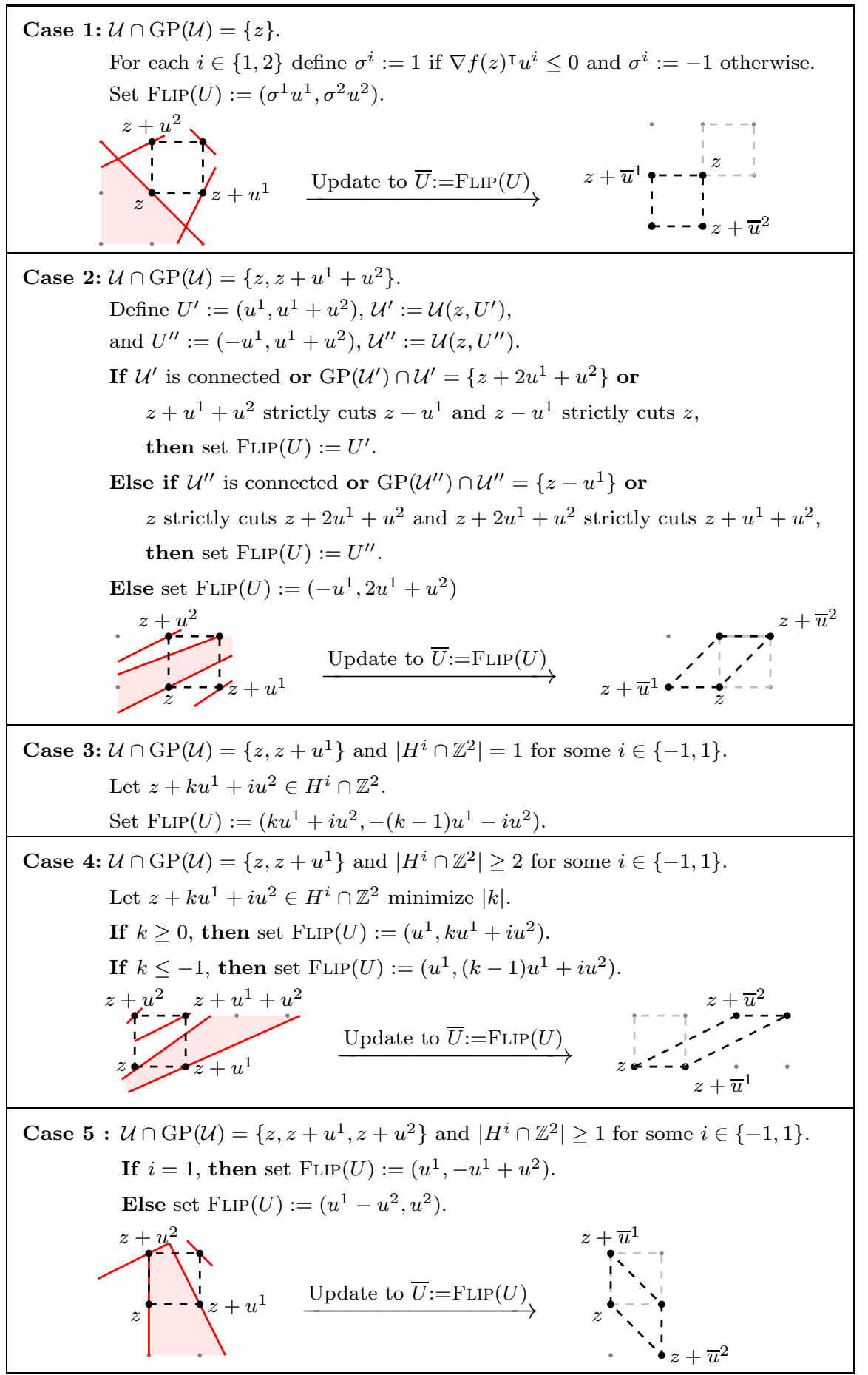

Table 1 The different replacements for $U$ used in $\operatorname{FLIP}(U)$. Sample updates are given with the convex hulls of $\mathcal{U}(z, U)$ and $\mathcal{U}(z, \bar{U})$ in dashed black lines and $\operatorname{GP}(\mathcal{U})$ in red. 
Lemma 3 Let $\mathcal{U}=\mathcal{U}(z, U)$ be connected and preprocessed as in Lemma 2 . Then $\operatorname{GP}(\mathcal{U})$ is lattice-free if and only if $H^{-1} \cap \mathbb{Z}^{2}=\emptyset$ and $H^{1} \cap \mathbb{Z}^{2}=\emptyset$.

Proof If $\operatorname{GP}(\mathcal{U})$ is lattice-free, then $H^{-1} \cap \mathbb{Z}^{2}$ and $H^{1} \cap \mathbb{Z}^{2}$ are empty. Assume to the contrary that $\operatorname{GP}(\mathcal{U})$ is not lattice-free but $H^{-1} \cap \mathbb{Z}^{2}=H^{1} \cap \mathbb{Z}^{2}=\emptyset$. Let $x \in \operatorname{intr}(\operatorname{GP}(\mathcal{U})) \cap \mathbb{Z}^{2}$ be of any vector of the form $x=z+k_{1} u^{1}+k_{2} u^{2}$, where $k_{1}, k_{2} \in \mathbb{Z}$ and such that $\left|k_{2}\right|$ is minimized. Note that $\left|k_{2}\right| \neq 1$ because $H^{-1} \cap \mathbb{Z}^{2}=H^{1} \cap \mathbb{Z}^{2}=\emptyset$. If $k_{2}=0$ and $k_{1}<0$, then $z$ is a convex combination of $x$ and $z+u^{1}$ and is contained in $\operatorname{intr}(\operatorname{GP}(\mathcal{U}))$, which is a contradiction. Similarly, if $k_{2}=0$ and $k_{1} \geq 1$, then $z+u^{1} \in \operatorname{intr}(\operatorname{GP}(\mathcal{U}))$. Hence, $\left|k_{2}\right| \geq 2$.

Both $z$ and $z+u^{1}$ are in $\operatorname{GP}(\mathcal{U})$ because $\mathcal{U}$ is connected. The set $\operatorname{conv}\{z, z+$ $\left.u^{1}, x\right\}$ is contained in $\operatorname{GP}(\mathcal{U})$ because $\operatorname{GP}(\mathcal{U})$ is convex. From the linearity of the determinant and the equation $|\operatorname{det}(U)|=1$, it follows that

$\left|\operatorname{det}\left(z-x,\left(z+u^{1}\right)-x\right)\right|=\left|\operatorname{det}\left(-k_{1} u^{1}-k_{2} u^{2},-\left(k_{1}-1\right) u^{1}-k_{2} u^{2}\right)\right|=\left|k_{2}\right| \geq 2$.

This implies that $\operatorname{conv}\left\{z, z+u^{1}, x\right\} \backslash\left\{z, z+u^{1}, x\right\}$ contains an integer vector $\bar{x}:=z+k_{1}^{\prime} u^{1}+k_{2}^{\prime} u^{2}$ with $k_{1}^{\prime}, k_{2}^{\prime} \in \mathbb{Z}$ (see, e.g., [7, Page 291, Corollary (2.6)]). There are no integer vectors in $\operatorname{conv}\left\{z, z+u^{1}\right\} \backslash\left\{z, z+u^{1}\right\}$ because $U$ is unimodular, so $0<\left|k_{2}^{\prime}\right|<\left|k_{2}\right|$. Hence, $\bar{x} \in \operatorname{intr}(\operatorname{GP}(\mathcal{U}))$, which contradicts the choice of $k_{2}$.

Lemma 1$]($ ii $)$ implies $1 \leq|\mathcal{U} \cap \operatorname{GP}(\mathcal{U})| \leq 4$. However, Table 1 does not consider $|\mathcal{U} \cap \operatorname{GP}(\mathcal{U})|=4$ because $\operatorname{GP}(\mathcal{U})$ is lattice-free in this setting.

Corollary 2 If $|\mathcal{U} \cap \operatorname{GP}(\mathcal{U})|=4$, then $\operatorname{GP}(\mathcal{U})$ is lattice-free.

Proof If $|\mathcal{U} \cap \mathrm{GP}(\mathcal{U})|=4$, then $\mathcal{U}$ is connected. Assume to the contrary that $z+$ $k u^{1}+u^{2} \in H^{1} \cap \mathbb{Z}^{2}$ for $k \geq 1$. Then $z+u^{1}+u^{2} \in \operatorname{conv}\left\{z+u^{2}, z+k u^{1}+u^{2}\right\}$, and Observation 1implies that $z+u^{1}+u^{2} \in \operatorname{intr}(\operatorname{GP}(\mathcal{U}))$, which is a contradiction. Using similar arguments, it can be shown that $H^{1} \cap \mathbb{Z}^{2}=H^{-1} \cap \mathbb{Z}^{2}=\emptyset$. Hence, $\operatorname{GP}(\mathcal{U})$ is lattice-free by Lemma 3 .

The previous results show that the cases in Table 1 suffice.

Lemma 4 Let $\mathcal{U}$ be preprocessed as in Lemma 2. If $\mathcal{U}$ does not fit into a case of Table 1, then $\operatorname{GP}(\mathcal{U})$ is lattice-free.

Proof If $\mathcal{U}$ is connected, then the conditions of Lemma 3 or Corollary 2 are met and $\operatorname{GP}(\mathcal{U})$ is lattice-free. Otherwise, $\mathcal{U}$ is disconnected and falls into a case of the table.

In the remainder of this section we consider the set $\operatorname{FLIP}(\mathcal{U})$ for every case in the table. For this analysis, we briefly elaborate on the two measures of progress to prove Theorem 1 (ii) and (iii). The first measure is the smallest function value in $\mathcal{U}$. Given another unimodular set $\mathcal{U}^{\prime}$, we say

$$
\mathcal{U}<_{f} \mathcal{U}^{\prime} \text { if } \min \{f(z): z \in \mathcal{U}\}<\min \left\{f(z): z \in \mathcal{U}^{\prime}\right\}
$$

We define $\mathcal{U} \leq{ }_{f} \mathcal{U}^{\prime}$ and $\mathcal{U}={ }_{f} \mathcal{U}^{\prime}$ similarly. Lemma 5 establishes the inequality $\operatorname{FLIP}(\mathcal{U}) \leq_{f} \mathcal{U}$ for every case. The proof follows directly from the definitions in the table and Lemma 1. 
Lemma 5 Let $\mathcal{U}$ be preprocessed as in Lemma 圆. Then $\mathcal{U} \cap \operatorname{GP}(\mathcal{U}) \subseteq \operatorname{FLIP}(\mathcal{U})$ and $\operatorname{FLIP}(\mathcal{U}) \leq_{f} \mathcal{U}$.

Our second measure of progress is (4). To motivate this, define the orthants corresponding to $\mathcal{U}=\mathcal{U}(z, U)$ :

$$
\begin{aligned}
O_{\mathcal{U}}(z) & :=\left\{z+U r: r \in \mathbb{R}_{\leq 0} \times \mathbb{R}_{\leq 0}\right\}, \\
O_{\mathcal{U}}\left(z+u^{1}\right) & :=\left\{\left(z+u^{1}\right)+U r: r \in \mathbb{R}_{\geq 0} \times \mathbb{R}_{\leq 0}\right\}, \\
O_{\mathcal{U}}\left(z+u^{2}\right) & :=\left\{\left(z+u^{2}\right)+U r: r \in \mathbb{R}_{\leq 0} \times \mathbb{R}_{\geq 0}\right\}, \text { and } \\
O_{\mathcal{U}}\left(z+u^{1}+u^{2}\right) & :=\left\{\left(z+u^{1}+u^{2}\right)+U r: r \in \mathbb{R}_{\geq 0} \times \mathbb{R}_{\geq 0}\right\} .
\end{aligned}
$$

See Figure 2 (i) for an example. The matrix $U$ is unimodular, so for every $x \in \mathbb{Z}^{2}$ and $w \in \mathcal{U}$ the difference vector $x-w$ is an integer combination of $\left\|U^{-1}(x-w)\right\|_{1}$ many signed copies of $u^{1}$ and $u^{2}$. Moreover, there is a unique $w^{*} \in \mathcal{U}$ such that $x \in O_{\mathcal{U}}\left(w^{*}\right)$, and this $w^{*}$ minimizes $\left\|U^{-1}(x-w)\right\|_{1}$ over $w \in \mathcal{U}$. Denote this minimum value by

$$
r_{\mathcal{U}}(x):=\min \left\{\left\|U^{-1}(x-w)\right\|_{1}: w \in \mathcal{U}\right\} .
$$

The value in (4) equals $\min \left\{r_{\mathcal{U}}\left(z^{*}\right): z^{*}\right.$ optimal for (피) $\}$. Note $r_{\mathcal{U}}(x)=0$ if and only if $x \in \mathcal{U}$. If

$$
\begin{aligned}
& \min \left\{r_{\mathcal{U}}\left(z^{*}\right): z^{*} \text { is optimal for }(\overline{\mathrm{CM}})\right\} \\
&<\min \left\{r_{\mathcal{U}^{\prime}}\left(z^{*}\right): z^{*} \text { is optimal for }(\overline{\mathrm{CM}})\right\},
\end{aligned}
$$

then we write $\mathcal{U}<_{r} \mathcal{U}^{\prime}$. We define $\mathcal{U} \leq_{r} \mathcal{U}^{\prime}$ and $\mathcal{U}={ }_{r} \mathcal{U}^{\prime}$ similarly. The minima in (8) exist due to Assumption (1).

The next result follows from the definition of $=_{r}$ and Lemmata [1] $(i)$ and 5

Lemma 6 If $\mathcal{U}$ contains a minimizer $z^{*}$ of (CM), then $z^{*} \in \operatorname{FLIP}(\mathcal{U})$ and $r_{\mathcal{U}}\left(z^{*}\right)=r_{\mathrm{FLIP}(\mathcal{U})}\left(z^{*}\right)=0$.

We now proceed to analyze the cases independently. In each subsection, we let $z^{*}$ denote an arbitrary optimal solution to (CM).

\subsection{An analysis of Case $\mathbf{1 .}$}

Lemma 7 Suppose $\mathcal{U}$ fits into Case 1. Then

(i) $\operatorname{FLIP}(\mathcal{U}) \leq_{r} \mathcal{U}$

(ii) if $\operatorname{FLIP}(\mathcal{U})={ }_{f} \mathcal{U}$ and $\operatorname{FLIP}(\mathcal{U})={ }_{r} \mathcal{U}$, then $\operatorname{FLIP}(\mathcal{U})$ is connected.

Proof First, we consider the outcome that

$\operatorname{FLip}(U)=\left(-u^{1},-u^{2}\right)$ and $\operatorname{FLip}(\mathcal{U})=\left\{z, z-u^{1}, z-u^{2}, z-u^{1}-u^{2}\right\}$.

See Figure 2. Here, $z$ strictly cuts $z+u^{1}$ and $z+u^{2}$. By Lemma 1 (iv) we have

$$
z \text { strictly cuts } z+k_{1} u^{1}+k_{2} u^{2} \quad \forall k_{1}, k_{2} \geq 0 \text { with } k_{1}+k_{2} \geq 1 \text {. }
$$


This implies $z^{*} \notin O_{\mathcal{U}}\left(z+u^{1}+u^{2}\right)$. Hence, $z^{*} \in O_{\mathcal{U}}(z) \cup O_{\mathcal{U}}\left(z+u^{1}\right) \cup O_{\mathcal{U}}\left(z+u^{2}\right)$. By (9)), if $z^{*} \in O_{\mathcal{U}}\left(z+u^{2}\right)$, then

$$
z^{*}=\left(z+u^{2}\right)+k^{1}\left(-u^{1}\right)+k_{2} u^{2}=\left(z-u^{1}\right)+\left(k_{1}-1\right)\left(-u^{1}\right)+\left(k_{2}+1\right) u^{2},
$$

where $k_{1} \geq 1$ and $k_{2} \geq 0$. This last expression implies that $z^{*} \in O_{\mathrm{FLIP}(\mathcal{U})}\left(z-u^{1}\right)$ and $r_{\operatorname{FLIP}(\mathcal{U})}\left(z^{*}\right)=k_{1}+k_{2}=r_{\mathcal{U}}\left(z^{*}\right)$. So, $\operatorname{FLIP}(\mathcal{U}) \leq_{r} \mathcal{U}$. We derive $\operatorname{FLIP}(\mathcal{U}) \leq_{r}$ $\mathcal{U}$ similarly if $z^{*} \in O_{\mathcal{U}}\left(z+u^{1}\right) \cup O_{\mathcal{U}}(z)$.

Suppose further that $\operatorname{FLIP}(\mathcal{U})={ }_{f} \mathcal{U}$ and $\operatorname{FLIP}(\mathcal{U})={ }_{r} \mathcal{U}$. We claim that $\operatorname{FLIP}(\mathcal{U})$ is connected. Here, we have $z \in \operatorname{GP}(\operatorname{FLIP}(\mathcal{U}))$, otherwise some vector in $\operatorname{FLIP}(\mathcal{U})$ strictly cuts $z$ and $\operatorname{FLIP}(\mathcal{U})<_{f} \mathcal{U}$ by Proposition 1 Furthermore, $z$ does not strictly cut any vector in $\operatorname{FLIP}(\mathcal{U})$ by (9). Assume to the contrary that $\operatorname{FLIP}(\mathcal{U})$ is disconnected. Then, $\operatorname{FLIP}(\mathcal{U}) \cap \operatorname{GP}(\mathcal{U})=\left\{z, z-u^{1}-u^{2}\right\}$ by Lemma 1(v) Lemma 1(iii) states $z-u^{1}-u^{2}$ strictly cuts $z-u^{1}$ and $z-u^{1}$. Thus, $z-u^{1}-u^{2}$ strictly cuts $z$ by Lemma 1 (iv), which is a contradiction.

The other outcomes in Case 1 are $\operatorname{FLIP}(U)=\left(-u^{1}, u^{2}\right)$ and $\operatorname{FLIP}(U)=$ $\left(u^{1},-u^{2}\right)$. These settings are symmetric, so suppose

$$
\operatorname{FLIP}(U)=\left(u^{1},-u^{2}\right) \text { and } \operatorname{FLip}(\mathcal{U})=\left\{z, z+u^{1}, z-u^{2}, z+u^{1}-u^{2}\right\} .
$$

Here, $z$ strictly cuts $z-u^{1}$ and $z+u^{2}$. Thus, by Lemma 1 (iv) $z$ strictly cuts every vector in $O_{\mathcal{U}}\left(z+u^{2}\right)$ and $z^{*} \notin O_{\mathcal{U}}\left(z+u^{2}\right)$. Since $z+u^{1}+u^{2} \notin \operatorname{GP}(\mathcal{U})$, $z+u^{1}+u^{2}$ is strictly cut by at least one vector in $\mathcal{U} \backslash\left\{z+u^{1}+u^{2}\right\}$. If $z+u^{1}+u^{2}$ is strictly cut by $z+u^{1}$ or $z+u^{2}$, then Lemma 1 (iv) implies that every vector in $O_{\mathcal{U}}\left(z+u^{1}+u^{2}\right)$ is strictly cut and hence $z^{*} \notin O_{\mathcal{U}}\left(z+u^{1}+u^{2}\right)$. Suppose that neither $z+u^{1}$ nor $z+u^{2}$ strictly cut $z+u^{1}+u^{2}$. This implies that $z+u^{2}$ does not strictly cut $z+u^{1}$. Since $z$ does not strictly cut $z+u^{1}$ by assumption, $z+u^{1}$ is strictly cut by $z+u^{1}+u^{2}$. Because $z+u^{1}+u^{2}$ does not strictly cut $z$, Lemma 1 (iv) yields that $z+u^{1}+u^{2}$ strictly cuts every vector of the form $\left(z+u^{1}+u^{1}\right)+\left(-k_{1}\right) u^{1}+\left(-k_{2}\right) u^{2}$ with $k_{1} \geq k_{2} \geq 0$. Similarly, since $z$ strictly cuts $z+u^{1}+u^{2}$ but does not strictly cut $z+u^{1}$, Lemma 1 (iv) yields that $z$ strictly cuts every vector of the form $\left(z+u^{1}+u^{1}\right)+\left(-k_{1}\right) u^{1}+\left(-k_{2}\right) u^{2}$ with $0 \leq k_{1} \leq k_{2}$. Overall every vector in $O_{\mathcal{U}}\left(z+u^{1}+u^{2}\right)$ is strictly cut and thus $z^{*} \notin O_{\mathcal{U}}\left(z+u^{1}+u^{2}\right)$.

Hence, $z^{*} \in O_{\mathcal{U}}(z) \cup O_{\mathcal{U}}\left(z+u^{1}\right)$. If $z^{*} \in O_{\mathcal{U}}(z)$, then

$$
z^{*}=z+k_{1}\left(-u^{1}\right)+k_{2}\left(-u^{2}\right),
$$

where $k_{1}, k_{2} \geq 0$. If $k_{2}=0$, then $z^{*} \in O_{\mathrm{FLIP}(\mathcal{U})}(z)$ and $r_{\mathrm{FLIP}(\mathcal{U})}\left(z^{*}\right)=k_{1}=$ $r_{\mathcal{U}}\left(z^{*}\right)$. If $k_{2} \geq 1$, then $z^{*} \in O_{\operatorname{FLIP}(\mathcal{U})}\left(z-u^{2}\right)$ and

$$
z^{*}=\left(z-u^{2}\right)+k_{1}\left(-u^{1}\right)+\left(k_{2}-1\right)\left(-u^{2}\right),
$$

which shows that $r_{\mathcal{U}}\left(z^{*}\right)=k_{1}+k_{2}>k_{1}+\left(k_{2}-1\right)=r_{\operatorname{FLIP}(\mathcal{U})}\left(z^{*}\right)$.

Symmetrically, if $z^{*} \in O_{\mathcal{U}}\left(z+u^{1}\right)$, then $r_{\operatorname{FLIP}(\mathcal{U})}\left(z^{*}\right) \leq_{r} r_{\mathcal{U}}\left(z^{*}\right)$. Furthermore, using a proof similar to above, we see that if $\operatorname{FLIP}(\mathcal{U})=_{f} \mathcal{U}$ and $\operatorname{FLIP}(\mathcal{U})={ }_{r} \mathcal{U}$, then $\operatorname{FLIP}(\mathcal{U})$ is connected. 


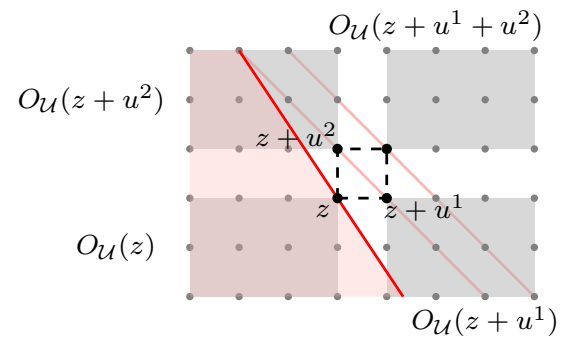

(i)

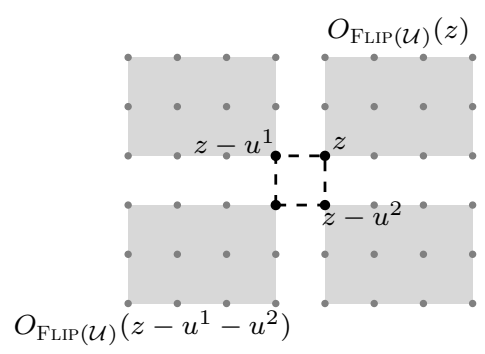

(ii)

Fig. 2 (i) Case 1 when $\operatorname{FLIP}(U)=\left(-u^{1},-u^{2}\right)$. $\operatorname{GP}(\mathcal{U})$ is also drawn. (ii) A sample update $\operatorname{FLIP}(U)=\left(-u^{1},-u^{2}\right)$.

A defining property of Case 1 is that $z \in \mathcal{U} \cap \operatorname{FLIP}(\mathcal{U})$. From this, we make the following observations for later use.

Lemma 8 If $\mathcal{U}$ fits into Case 1, then $\min \{f(v): v \in \mathcal{U}\}=f(z)$. Also, either $\operatorname{FLIP}(\mathcal{U})$ is connected or $z$ is strictly cut by a vector in $\operatorname{FLIP}(\mathcal{U})$.

Proof The fact that $z$ minimizes $f$ over $\mathcal{U}$ follows from Proposition 1 because each vector in $\mathcal{U} \backslash\{z\}$ is strictly cut by some vector in $\mathcal{U}$. If $z$ is not strictly cut by a vector in $\operatorname{FLIP}(\mathcal{U})$, then $z \in \operatorname{GP}(\operatorname{FLIP}(\mathcal{U}))$ and $|\operatorname{FLIP}(\mathcal{U}) \cap \operatorname{GP}(\operatorname{FLIP}(\mathcal{U}))| \geq$ 2 by Lemma $1(v)$. Assume to the contrary that $\operatorname{FLIP}(\mathcal{U})$ is disconnected. Thus, $\operatorname{FLIP}(\mathcal{U})=\left\{z, z+\sigma^{1} u^{1}+\sigma^{2} u^{2}\right\}$, where $\sigma^{1}, \sigma^{2}$ are defined in Table1, The vector $z+\sigma^{1} u^{1}+\sigma^{2} u^{2}$ must strictly cut $z+\sigma^{1} u^{1}, z+\sigma^{2} u^{2} \in \operatorname{FLIP}(\mathcal{U})$. Then Lemma1 (iv) implies that $z+\sigma^{1} u^{1}+\sigma^{2} u^{2}$ strictly cuts $z$, which is a contradiction.

\subsection{An analysis of Case 2 .}

The following sets are helpful to analyze Case 2

$$
\begin{aligned}
O_{\mathcal{U}}\left(z+u^{1}+u^{2}\right)_{B} & :=\left\{\left(z+u^{1}+u^{2}\right)+k_{1}\left(u^{1}+u^{2}\right)+k_{2} u^{2}: k_{1}, k_{2} \in \mathbb{R}_{\geq 0}\right\}, \\
O_{\mathcal{U}}\left(z+u^{1}+u^{2}\right)_{A} & :=O_{\mathcal{U}}\left(z+u^{1}+u^{2}\right) \backslash O_{\mathcal{U}}\left(z+u^{1}+u^{2}\right)_{B}, \\
O_{\mathcal{U}}(z)_{B} & :=\left\{z+k_{1}\left(u^{1}+u^{2}\right)+k_{2} u^{2}: k_{1}, k_{2} \in \mathbb{R}_{\leq 0}\right\}, \text { and } \\
O_{\mathcal{U}}(z)_{A} & :=O_{\mathcal{U}}(z) \backslash O_{\mathcal{U}}(z)_{B} .
\end{aligned}
$$

Figure 3 (i) illustrates these sets.

\section{Lemma 9 Suppose $\mathcal{U}$ fits into Case 2, Then}

(i) $\operatorname{FLIP}(\mathcal{U}) \leq_{r} \mathcal{U}$.

(ii) if $\operatorname{FLIP}(U)=\left(-u^{1}, 2 u^{1}+u^{2}\right)$ and $\mathcal{U}$ does not contain a minimizer of (CM), then $\operatorname{FLIP}(\mathcal{U})<_{r} \mathcal{U}$.

(iii) if $\operatorname{FLIP}(\mathcal{U})={ }_{f} \mathcal{U}$ and $\operatorname{FLIP}(\mathcal{U})={ }_{r} \mathcal{U}$, then $\operatorname{FLIP}(\mathcal{U})$ is connected.

Proof First, we prove $\operatorname{FLIP}(\mathcal{U}) \leq_{r} \mathcal{U}$. By Lemma $[$ we assume that $\mathcal{U}$ does not contain a minimizer $z^{*}$ of (CM). By Lemma $1(i), z^{*} \in \operatorname{intr}(\operatorname{GP}(\mathcal{U}))$. 


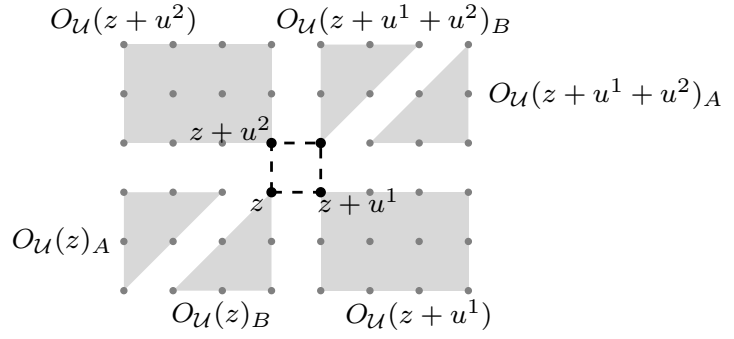

(i)

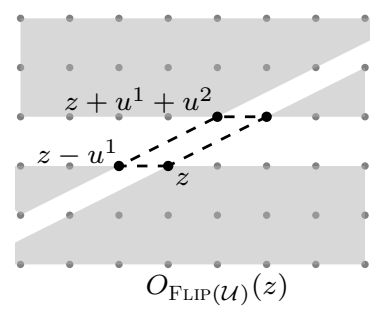

(ii)

Fig. 3 (i) Sets used to analyze Case 2 (ii) A sample update for the third outcome of Case $22 \operatorname{Flip}(U)=\left(-u^{1}, 2 u^{1}+u^{2}\right)$.

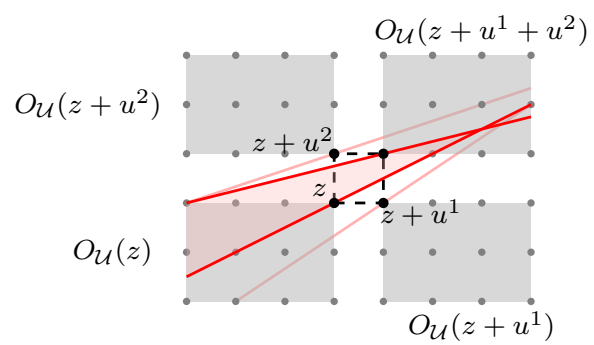

(i)

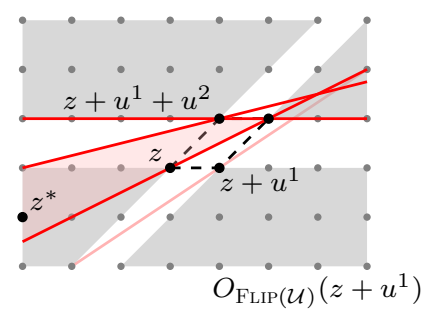

(ii)

Fig. 4 (i) Case 2 where $\operatorname{FLIP}(\mathcal{U})=\mathcal{U}^{\prime} . \operatorname{GP}(\mathcal{U})$ is also drawn. (ii) A sample update $\operatorname{FLIP}(\mathcal{U})=\mathcal{U}^{\prime} . \operatorname{GP}\left(\mathcal{U}^{\prime}\right)$ is also drawn. Note that $\operatorname{GP}\left(\mathcal{U}^{\prime}\right)$ is connected.

If $z^{*} \in O_{\mathcal{U}}\left(z+u^{2}\right)$, then $z+u^{2} \in \operatorname{conv}\left\{z^{*}, z+u^{1}+u^{2}, z\right\}$ giving the contradiction $z+u^{2} \in \operatorname{intr}(\operatorname{GP}(\mathcal{U}))$. By symmetry, $z^{*} \notin O_{\mathcal{U}}\left(z+u^{1}\right)$. The preprocessing in Lemma 2 states that $z+u^{1}+u^{2}$ strictly cuts $z+u^{2}$ and does not cut $z+u^{1}$. Thus, $z+u^{1}+u^{2}$ strictly cuts every vector in $O_{\mathcal{U}}\left(z+u^{1}+u^{2}\right)_{B}$ by Lemma $1(i v)$. So, $z^{*} \notin O_{\mathcal{U}}\left(z+u^{1}+u^{2}\right)_{B}$. Similarly, $z^{*} \notin O_{\mathcal{U}}(z)_{B}$. Therefore, $z^{*} \in O_{\mathcal{U}}(z)_{A} \cup O_{\mathcal{U}}\left(z+u^{1}+u^{2}\right)_{A}$. As implied by Figure 3 (i), the sets $O_{\mathcal{U}}(z)_{A}$ and $O_{\mathcal{U}}\left(z+u^{1}+u^{2}\right)_{A}$ are symmetric around $\mathcal{U}$. Hence, we simplify the proof of $\operatorname{FLIP}(\mathcal{U}) \leq_{r} \mathcal{U}$ by assuming without loss of generality that $z^{*} \in O_{\mathcal{U}}(z)_{A}$.

Case 2 has three outcomes. The first two update $U$ to one of the matrices $U^{\prime}$ and $U^{\prime \prime}$ from Table 1. The proofs of correctness for each of these outcomes are similar. We prove $\operatorname{FLIP}(\mathcal{U}) \leq_{r} \mathcal{U}$ when

$$
\begin{aligned}
\operatorname{FLip}(U) & =U^{\prime}=\left(u^{1}, u^{1}+u^{2}\right) \\
\text { and } \operatorname{FLip}(\mathcal{U}) & =\mathcal{U}^{\prime}=\left\{z, z+u^{1}, z+u^{1}+u^{2}, z+2 u^{1}+u^{2}\right\} .
\end{aligned}
$$

See Figure 4. Here we have

$$
z^{*}=z+k_{1}\left(-u^{1}\right)+k_{2}\left(-u^{2}\right)=z+\left(k_{1}-k_{2}\right) u^{1}+k_{2}\left(-u^{1}-u^{2}\right),
$$

where $k_{1}, k_{2} \geq 0$ and $k_{1} \geq k_{2}+1$. Thus,

$$
r_{\mathcal{U}}\left(z^{*}\right)=k_{1}+k_{2} \geq\left(k_{1}-k_{2}\right)+k_{2}=r_{\mathrm{FLIP}(\mathcal{U})}\left(z^{*}\right),
$$


and $\operatorname{FLIP}(\mathcal{U}) \leq_{r} \mathcal{U}$

Next, we consider the third outcome of Case 2

$\operatorname{FLIP}(U)=\left(-u^{1}, 2 u^{1}+u^{2}\right)$ and $\operatorname{FLIP}(\mathcal{U})=\left\{z, z-u^{1}, z+u^{1}+u^{2}, z+2 u^{1}+u^{2}\right\}$.

In this setting $z^{*} \in O_{\mathrm{FLIP}(\mathcal{U})}\left(z-u^{1}\right) \cup O_{\mathrm{FLIP}(\mathcal{U})}(z)$. This is indicated in Figure 3, The proof when $z^{*} \in O_{\mathrm{FLIP}(\mathcal{U})}(z)$ is similar to when $z^{*} \in O_{\mathrm{FLIP}(\mathcal{U})}\left(z-u^{1}\right)$, so we consider $z^{*} \in O_{\mathrm{FLIP}(\mathcal{U})}(z)$. There exist $k_{1}, k_{2} \in \mathbb{Z}_{\geq 0}$ such that

$$
z^{*}=z+k_{1}\left(-u^{1}\right)+k_{2}\left(-u^{2}\right)=z+\left(-k_{1}+2 k_{2}\right) u^{1}+k_{2}\left(-2 u^{1}-u^{2}\right) .
$$

We have $k_{1}>k_{2}$ because $z^{*} \in O_{\mathcal{U}}(z)_{A}$. This is equivalent to $k_{2}>\left(-k_{1}+2 k_{2}\right)$. Thus,

$$
r_{\mathcal{U}}\left(z^{*}\right)=k_{1}+k_{2}>\left(-k_{1}+2 k_{2}\right)+k_{2}=r_{\mathrm{FLIP}(\mathcal{U})}\left(z^{*}\right)
$$

and $\operatorname{FLIP}(\mathcal{U})<_{r} \mathcal{U}$. This proves $(i)$ and $(i i)$

Now, suppose that $\operatorname{FLIP}(\mathcal{U})={ }_{f} \mathcal{U}$ and $\operatorname{FLIP}(\mathcal{U})={ }_{r} \mathcal{U}$ and $\operatorname{FLIP}(\mathcal{U})=\mathcal{U}^{\prime}$ or $\operatorname{FLIP}(\mathcal{U})=\mathcal{U}^{\prime \prime}$. We give the proof when $\operatorname{FLIP}(\mathcal{U})=\mathcal{U}^{\prime}$ as the other proof is similar. The definition of $\operatorname{FLIP}(\mathcal{U})=\mathcal{U}^{\prime}$ in Table 1 implies that one of three things must occur: $\mathcal{U}^{\prime}$ is connected, $\operatorname{GP}\left(\mathcal{U}^{\prime}\right) \cap \mathcal{U}^{\prime}=\left\{z+2 u^{1}+u^{2}\right\}$, or $z+u^{1}+u^{2}$ strictly cuts $z-u^{1}$ and $z-u^{1}$ strictly cuts $z$. If $\mathcal{U}^{\prime}$ is connected, then (iii) holds. If $\mathcal{U}^{\prime} \cap \operatorname{GP}\left(\mathcal{U}^{\prime}\right)=\left\{z+2 u^{1}+u^{2}\right\}$, then $\mathcal{U}^{\prime}<_{f} \mathcal{U}$, which contradicts $\operatorname{FLIP}(\mathcal{U})={ }_{f} \mathcal{U}$.

It remains to consider when $z+u^{1}+u^{2}$ strictly cuts $z-u^{1}$ and $z-u^{1}$ strictly cuts $z$. Here, $f\left(z+u^{1}+u^{2}\right)<f(z)$ and $f\left(z+u^{1}+u^{2}\right)=\min \{f(w): w \in \mathcal{U}\}$. Moreover, $f\left(z+u^{1}+u^{2}\right)=\min \left\{f(w): w \in \mathcal{U}^{\prime}\right\}$ because $\operatorname{FLIP}(\mathcal{U})={ }_{f} \mathcal{U}$. Thus, $z+u^{1}+u^{2} \in \mathcal{U}^{\prime} \cap \operatorname{GP}\left(\mathcal{U}^{\prime}\right)$ by Proposition 1. The definition of Case 2 and the preprocessing in Lemma 2 imply that $z+u^{1}+u^{2}$ does not strictly cut any vector in $\mathcal{U}^{\prime} \backslash\left\{z+u^{1}+u^{2}\right\}$. By Lemma 1 $1(v)$ we have $\left|\mathcal{U}^{\prime} \cap \operatorname{GP}\left(\mathcal{U}^{\prime}\right)\right| \geq 2$. If $\mathcal{U}^{\prime}$ was disconnected, then $\mathcal{U}^{\prime} \cap \operatorname{GP}\left(\mathcal{U}^{\prime}\right)=\left\{z+u^{1}+u^{2}, z+u^{1}\right\}$. However, $z+u^{1} \notin \mathcal{U}^{\prime} \cap \operatorname{GP}\left(\mathcal{U}^{\prime}\right)$ because it is strictly cut by $z$ by the preprocessing on $\mathcal{U}$. Hence, $\mathcal{U}^{\prime}$ is connected. In fact, we have shown the stronger statement that if $\operatorname{FLIP}(\mathcal{U})={ }_{f} \mathcal{U}, \operatorname{FLIP}(\mathcal{U})={ }_{r} \mathcal{U}$, and $f\left(z+u^{1}+u^{2}\right) \leq f(z)$, then $\mathcal{U}^{\prime}$ is connected

It is left to prove $($ iii $)$ when $\operatorname{FLIP}(\mathcal{U})={ }_{f} \mathcal{U}, \operatorname{FLIP}(\mathcal{U})={ }_{r} \mathcal{U}$, and $\operatorname{FLIP}(U)=$ $\left(-u^{1}, 2 u^{1}+u^{2}\right)$. We claim that this cannot occur. By (ii) it must be the case that $\mathcal{U} \cap \operatorname{GP}(\mathcal{U})=\left\{z, z+u^{1}+u^{2}\right\}$ contains a minimizer of (CM). The set $\left\{z, z+u^{1}+u^{2}\right\}$ is contained in $\mathcal{U}^{\prime} \cap \mathcal{U}^{\prime \prime}$. Hence, $\mathcal{U}={ }_{f} \mathcal{U}^{\prime}={ }_{f} \mathcal{U}^{\prime \prime}$ and $\mathcal{U}={ }_{r}$ $\mathcal{U}^{\prime}={ }_{r} \mathcal{U}^{\prime \prime}$. We demonstrated in the previous paragraph that $\mathcal{U}^{\prime}$ is connected if $f\left(z+u^{1}+u^{2}\right) \leq f(z)$ and that $\mathcal{U}^{\prime \prime}$ is connected if $f(z) \leq f\left(z+u^{1}+u^{2}\right)$. Hence, $\operatorname{FLIP}(U)$ will not equal $\left(-u^{1}, 2 u^{1}+u^{2}\right)$ in this setting but rather $\operatorname{FLIP}(\mathcal{U})=\mathcal{U}^{\prime}$ or $\operatorname{FLIP}(\mathcal{U})=\mathcal{U}^{\prime \prime}$.

Unlike the connected cases, gradient information does not seem sufficient to make a precise flip in Case 2 without 'guessing'. This guessing is the cause of the more involved subcases. The necessity of the three conditions for $\operatorname{FLIP}(\mathcal{U})=\mathcal{U}^{\prime}$ or $\operatorname{FLIP}(\mathcal{U})=\mathcal{U}^{\prime \prime}$ is reflected in the next result. 


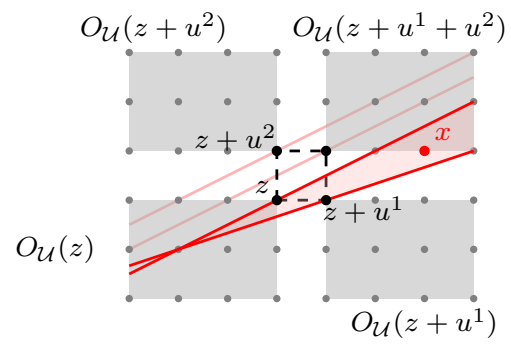

(i)

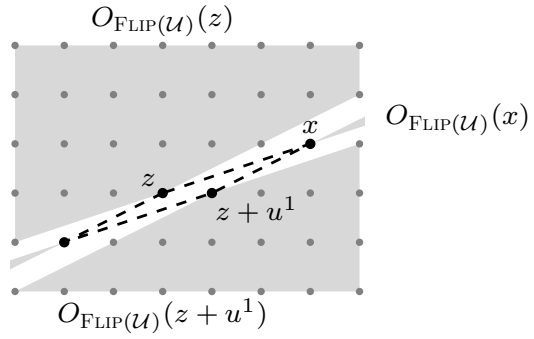

(ii)

Fig. 5 (i) The orthants used to prove Theorem 3in Case 3 GP(U) is also drawn. (ii) The orthants of $\operatorname{FLIP}(\mathcal{U})$ when $\left|H^{1} \cap \operatorname{GP}(\mathcal{U})\right|=1$.

Lemma 10 Assume $\mathcal{U}$ fits into Case 2 and let $w^{*}$ minimize $f(v)$ over $\mathcal{U}$. If $\operatorname{FLIP}(\mathcal{U})=\mathcal{U}^{\prime}$ or $\operatorname{FLIP}(\mathcal{U})=\mathcal{U}^{\prime \prime}$, then $\operatorname{FLIP}(\mathcal{U})$ is connected or $w^{*}$ is strictly cut by a vector in $\operatorname{FLIP}(\mathcal{U})$.

Proof The outcomes $\operatorname{FLIP}(\mathcal{U})=\mathcal{U}^{\prime}$ or $\operatorname{FLIP}(\mathcal{U})=\mathcal{U}^{\prime \prime}$ are symmetric, so we assume

$$
\operatorname{FLIP}(\mathcal{U})=\mathcal{U}^{\prime}=\left\{z, z+u^{1}, z+u^{1}+u^{2}, z+2 u^{1}+u^{2}\right\}
$$

The definition of $\operatorname{FLIP}(\mathcal{U})=\mathcal{U}^{\prime}$ in Table 1 implies that one of three things must occur: $\mathcal{U}^{\prime}$ is connected, $\operatorname{GP}\left(\mathcal{U}^{\prime}\right) \cap \mathcal{U}^{\prime}=\left\{z+2 u^{1}+u^{2}\right\}$, or $z+u^{1}+u^{2}$ strictly cuts $z-u^{1}$ and $z-u^{1}$ strictly cuts $z$. The first two conditions imply that the lemma holds. So, assume that $z+u^{1}+u^{2}$ strictly cuts $z-u^{1}$ and $z-u^{1}$ strictly cuts $z$.

This sequence of strict cuts implies $w^{*}=z+u^{1}+u^{2}$. If $z+u^{1}+u^{2} \notin$ $\mathcal{U}^{\prime} \cap \operatorname{GP}\left(\mathcal{U}^{\prime}\right)$, then the lemma holds. So, assume $z+u^{1}+u^{2} \in \mathcal{U}^{\prime} \cap \operatorname{GP}\left(\mathcal{U}^{\prime}\right)$. Then, $z+u^{1}+u^{2}$ is not strictly cut by any vector in $\mathcal{U}^{\prime}$. By the preprocessing of Case 2 2 the vector $z+u^{1}+u^{2}$ strictly cuts $z+u^{2}$, so it does not cut $z+2 u^{1}+u^{2}$. Also, $z+u^{1}+u^{2}$ does not strictly cut $z$ or $z+u^{1}$. Lemma $1(v)$ then implies $\left|\operatorname{GP}\left(\mathcal{U}^{\prime}\right) \cap \mathcal{U}^{\prime}\right| \geq 2$. One vector in $\operatorname{GP}\left(\mathcal{U}^{\prime}\right) \cap \mathcal{U}^{\prime}$ is $z+u^{1}+u^{2}$. The vector $z+u^{1}$ is strictly cut by $z$ because $\mathcal{U}$ falls into Case 2 , so the second vector in $\operatorname{GP}\left(\mathcal{U}^{\prime}\right) \cap \mathcal{U}^{\prime}$ must be $z$ or $z+2 u^{1}+u^{2}$. Hence, $\mathcal{U}^{\prime}$ is connected.

\subsection{An analysis of Case 3.}

Case 3 is executed if $\left|H^{-1} \cap \mathbb{Z}^{2}\right|=1$ or $\left|H^{1} \cap \mathbb{Z}^{2}\right|=1$. The two cases are symmetric, so we often assume $\left|H^{1} \cap \mathbb{Z}^{2}\right|=1$. See Figure 5 .

Lemma 11 Suppose $\mathcal{U}$ fits into Case 3. Then

(i) $\operatorname{FLIP}(\mathcal{U}) \leq_{r} \mathcal{U}$.

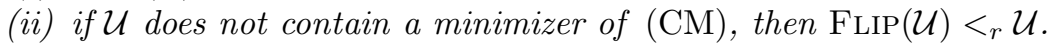

(iii) if $\operatorname{FLIP}(\mathcal{U})={ }_{f} \mathcal{U}$ and $\operatorname{FLIP}(\mathcal{U})={ }_{r} \mathcal{U}$, then $\operatorname{FLIP}(\mathcal{U})$ is connected and $\operatorname{FLIP}(\mathcal{U}) \cap \operatorname{GP}(\operatorname{FLIP}(\mathcal{U})) \backslash \mathcal{U} \neq \emptyset$. 
Proof First we show that $\operatorname{FLIP}(\mathcal{U}) \leq_{r} \mathcal{U}$. By Lemma 6 we assume that $\mathcal{U}$ does not contain a minimizer $z^{*}$ of (CM). By Lemma $1(i), z^{*} \in \operatorname{intr}(\operatorname{GP}(\mathcal{U}))$.

Using symmetry, we assume without loss of generality that $H^{1} \cap \mathbb{Z}^{2}=\{x\}$ and $x:=z+k u^{1}+u^{2}$ for $k \geq 2$. The setting when $k \leq-1$ is also symmetric. Here we have

$$
\begin{aligned}
& \operatorname{FLIP}(U)=\left(k u^{1}+u^{2},-(k-1) u^{1}-u^{2}\right) \text { and } \\
& \operatorname{FLIP}(\mathcal{U})=\left\{z, x, z+u^{1}, z-(k-1) u^{1}-u^{2}\right\} .
\end{aligned}
$$

If $z^{*} \in O_{\mathcal{U}}\left(z+u^{1}\right)$, then $z+u^{1} \in \operatorname{conv}\left\{z^{*}, z, x\right\}$ yielding the contradiction $z+u^{1} \in \operatorname{intr}(\operatorname{GP}(\mathcal{U}))$. A similar argument shows $z^{*} \notin O_{\mathcal{U}}\left(z+u^{2}\right)$. Hence, $z^{*} \in O_{\mathcal{U}}(z) \cup O_{\mathcal{U}}\left(z+u^{1}+u^{2}\right)$. The cases are symmetric, so we assume $z^{*} \in$ $O_{\mathcal{u}}\left(z+u^{1}+u^{2}\right)$. Thus,

$$
z^{*}=\left(z+u^{1}+u^{2}\right)+k_{1} u^{1}+k_{2} u^{2}
$$

where $k_{1}, k_{2} \geq 0$ and $k_{1}+k_{2} \geq 1$. If $z^{*} \in O_{\operatorname{FLIP}(\mathcal{U})}(z) \cap O_{\mathcal{U}}\left(z+u^{1}+u^{2}\right)$, then $x-u^{1} \in \operatorname{conv}\left\{z, x, z^{*}\right\}$ yielding the contradiction $x-u^{1} \in \operatorname{intr}(\operatorname{GP}(\mathcal{U}))$. Similarly, $z^{*} \notin O_{\mathrm{FLIP}(\mathcal{U})}\left(z+u^{1}\right) \cap O_{\mathcal{U}}\left(z+u^{1}+u^{2}\right)$ otherwise $x+u^{1} \in \operatorname{intr}(\operatorname{GP}(\mathcal{U}))$. Hence, $z^{*} \in\left[O_{\operatorname{FLIP}(\mathcal{U})}(x) \cup O_{\mathrm{FLIP}(\mathcal{U})}\left(z-(k-1) u^{1}-u^{2}\right)\right] \cap O_{\mathcal{U}}\left(z+u^{1}+u^{2}\right)$. We provide the analysis when $z^{*} \in O_{\operatorname{FLIP}(\mathcal{U})}(x) \cap O_{\mathcal{U}}\left(z+u^{1}+u^{2}\right)$ as the other case is similar. Here, $z^{*}$ can be rewritten as

$$
x+\left(k_{1}+k_{2}+1-k-k_{2} k\right)\left(k u^{1}+u^{2}\right)+\left(k+k_{2} k-1-k_{1}\right)\left((k-1) u^{1}+u^{2}\right),
$$

and $k_{1}+k_{2}+1-k-k_{2} k$ and $k+k_{2} k-1-k_{1}$ are nonnegative. Thus,

$$
r_{\mathcal{U}}\left(z^{*}\right)=k_{1}+k_{2}>k_{2}=r_{\mathrm{FLIP}(\mathcal{U})}\left(z^{*}\right)
$$

and $\operatorname{FLIP}(\mathcal{U})<_{r} \mathcal{U}$.

Finally, suppose that $\operatorname{FLIP}(\mathcal{U})={ }_{f} \mathcal{U}$. Lemma 5 and the equation $\operatorname{FuIP}(\mathcal{U})={ }_{f}$ $\mathcal{U}$ imply that $\left\{z, z+u^{1}\right\} \cap \operatorname{FLIP}(\mathcal{U}) \cap \operatorname{GP}(\operatorname{FLIP}(\mathcal{U})) \neq \emptyset$. We show that $x$ or $z-(k-1) u^{1}-u^{2}$ is in $\operatorname{GP}(\operatorname{FLIP}(\mathcal{U}))$, which will prove (iii). By Proposition 1 it is enough to show that $z$ and $z+u^{1}$ do not cut any vector in $\operatorname{FLIP}(\mathcal{U})$. The definition of $\operatorname{FLIP}(\mathcal{U})$ in Table 1 implies that $z$ and $z+u^{1}$ do not cut each other, and they do not cut $x$. The vectors $x-u^{1}$ and $x+u^{1}$ are cut by vectors in $\mathcal{U}$ because Case $\mathbf{3}$ is executed (as opposed to say Case 4). The vectors $z+u^{2}$ and $z+u^{1}+u^{2}$ do not cut any vectors of the form $z+r u^{1}+u^{2}$ for $r \geq 0$, otherwise they cut $x$ and Case 3 would not be executed. Hence, $x-u^{1}$ and $x+u^{1}$ are cut by $z$ and $z+u^{1}$. If $z$ strictly cuts $x+u^{1}$ but not $x$, then it also strictly cuts $z+u^{1}$, contradiction. Thus, $z+u^{1}$ cuts $x+u^{1}$, which implies that $z+u^{1}$ does not cut $z-(k-1) u^{1}-u^{2}$. Similarly, $z$ cuts $x-u^{1}$ and does not cut $z-(k-1) u^{1}-u^{2}$. 


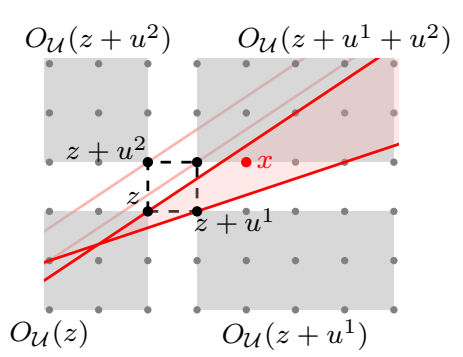

(i)

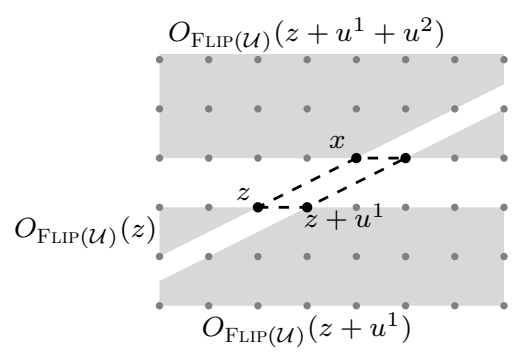

(ii)

Fig. 6 (i) The orthants used to prove Theorem 3 in Case 4 GP $(\mathcal{U})$ is also drawn. (ii) The orthants of $\operatorname{FLIP}(\mathcal{U})$ when $\left|H^{1} \cap \operatorname{GP}(\mathcal{U})\right| \geq 2$.

\subsection{An analysis of Case 4.}

Case 4 is executed if $\left|H^{-1} \cap \mathbb{Z}^{2}\right| \geq 2$ or $\left|H^{1} \cap \mathbb{Z}^{2}\right| \geq 2$. The two cases are symmetric, so we always assume $\left|H^{1} \cap \mathbb{Z}^{2}\right| \geq 2$. Furthermore, we assume that the vector $x:=z+k u^{1}+u^{2} \in H^{1} \cap \mathbb{Z}^{2}$ minimizing $|k|$ satisfies $k \geq 2$; if $k \leq-1$, then similar arguments can be made. Here, we have

$$
\operatorname{FLIP}(U)=\left(u^{1}, x\right) \text { and } \operatorname{FLIP}(\mathcal{U})=\left\{z, x, z+u^{1}, x+u^{1}\right\}
$$

Figure 6 illustrates this case.

Lemma 12 Suppose $\mathcal{U}$ fits into Case 4. Then

(i) $\operatorname{FLIP}(\mathcal{U}) \leq_{r} \mathcal{U}$

(ii) if $\mathcal{U}$ does not contain a minimizer of (त्CM), then $\operatorname{FLIP}(\mathcal{U})<_{r} \mathcal{U}$.

(iii) if $\operatorname{FLIP}(\mathcal{U})={ }_{f} \mathcal{U}$ and $\operatorname{FLIP}(\mathcal{U})={ }_{r} \mathcal{U}$, then $\operatorname{FLIP}(\mathcal{U})$ is connected and $\operatorname{FLIP}(\mathcal{U}) \cap \operatorname{GP}(\operatorname{FLIP}(\mathcal{U})) \backslash \mathcal{U} \neq \emptyset$.

Proof By symmetry we assume (10), where $x:=z+k u^{1}+u^{2} \in H^{1} \cap \mathbb{Z}^{2}$ minimizing $|k|$ satisfies $k \geq 2$. Using the definition of Case 4, we have $x-u^{1} \notin$ $\operatorname{intr}(\operatorname{GP}(\mathcal{U}))$.

First, we show that $\operatorname{FLIP}(\mathcal{U}) \leq_{r} \mathcal{U}$. By Lemma 6 we assume that $\mathcal{U}$ does not contain a minimizer $z^{*}$ of (CM). By Lemma [1] $(i)$, $z^{*} \in \operatorname{intr}(\operatorname{GP}(\mathcal{U}))$. If $z^{*} \in O_{\mathcal{U}}\left(z+u^{1}\right)$, then $z+u^{1} \in \operatorname{conv}\left\{z^{*}, z, x\right\}$ yielding the contradiction $z+u^{1} \in \operatorname{intr}(\operatorname{GP}(\mathcal{U}))$. If $z^{*} \in O_{\mathcal{U}}\left(z+u^{2}\right)$, then $z+u^{2} \in \operatorname{conv}\left\{z^{*}, z, x\right\}$ yielding the contradiction $z+u^{2} \in \operatorname{GP}(\mathcal{U})$. If $z^{*} \in O_{\mathcal{U}}(z)$, then $z^{*} \in O_{\mathrm{FLIP}(\mathcal{U})}(z) \cup$ $O_{\operatorname{FuIP}(\mathcal{U})}\left(z+u^{1}\right)$. This implies that either $z \in \operatorname{conv}\left\{z^{*}, z+u^{1}, x\right\}$ and $z \in$ $\operatorname{intr}(\operatorname{GP}(\mathcal{U}))$, or $z+u^{1} \in \operatorname{conv}\left\{z^{*}, z, x+u^{1}\right\}$ and $z+u^{1} \in \operatorname{intr}(\operatorname{GP}(\mathcal{U}))$. Both conclusions are contradictions, so $z^{*} \notin O_{\mathcal{U}}(z)$. Thus, $z^{*} \in O_{\mathcal{U}}\left(z+u^{1}+u^{2}\right)$ and it can be written as

$$
z^{*}=\left(z+u^{1}+u^{2}\right)+k_{1} u^{1}+k_{2} u^{2}
$$

where $k_{1}, k_{2} \geq 0$ and $k_{1}+k_{2} \geq 1$. If $k_{1} \leq k_{2}$, then $z+u^{1}+u^{2} \in \operatorname{conv}\left\{z^{*}, z, x\right\}$ yielding the contradiction $z+u^{1}+u^{2} \in \operatorname{GP}(\mathcal{U})$. Hence, $k_{1} \geq k_{2}+1$. The 
inclusion $z^{*} \in O_{\mathcal{U}}\left(z+u^{1}+u^{2}\right)$ implies that $z^{*} \in O_{\mathrm{FLIP}(\mathcal{U})}(x) \cup O_{\mathrm{FLIP}(\mathcal{U})}\left(x+u^{1}\right)$ (see Figure 6). We consider both settings.

If $z^{*} \in O_{\mathrm{FLIP}}(\mathcal{U})(x)$, then write

$$
z^{*}=x+\left(k\left(k_{2}+1\right)-k_{1}-1\right)\left(-u^{1}\right)+k_{2}\left(k u^{1}+u^{2}\right) .
$$

The vector $x-u^{1}$ can be written as

$$
\frac{k_{2}}{k\left(k_{2}+1\right)-k_{1}-1} \cdot z+\frac{k\left(k_{2}+1\right)-k_{1}-k_{2}-2}{k\left(k_{2}+1\right)-k_{1}-1} \cdot x+\frac{1}{k\left(k_{2}+1\right)-k_{1}-1} \cdot z^{*} .
$$

Hence, $k\left(k_{2}+1\right)-k_{1}-k_{2}-2<0$ otherwise $x-u^{1} \in \operatorname{conv}\left\{z, x, z^{*}\right\}$ yielding the contradiction $x-u^{1} \in \operatorname{intr}(\operatorname{GP}(\mathcal{U}))$. This inequality is equivalent to $k_{1}+k_{2}>$ $k\left(k_{2}+1\right)-2$. Thus,

$$
r_{\mathcal{U}}\left(z^{*}\right)=k_{1}+k_{2}>k\left(k_{2}+1\right)-2 \geq k\left(k_{2}+1\right)-k_{1}-1=r_{\mathrm{FLIP}(\mathcal{U})}\left(z^{*}\right) .
$$

Hence, $\operatorname{FLIP}(\mathcal{U})<_{r} \mathcal{U}$.

If $z^{*} \in O_{\operatorname{FLIP}(\mathcal{U})}\left(x+u^{1}\right)$, then rearrange (111) to write $z^{*}$ as

$$
z^{*}=\left(x+u^{1}\right)+\left(k_{1}-k_{2} k-k\right) u^{1}+k_{2}\left(k u_{1}+u_{2}\right) .
$$

Thus,

$$
r_{\mathcal{U}}\left(z^{*}\right)=k_{1}+k_{2}>\left(k_{1}-k_{2} k-k\right)+k_{2}=r_{\mathrm{FLIP}(\mathcal{U})}\left(z^{*}\right) .
$$

Hence, $\operatorname{FLIP}(\mathcal{U})<_{r} \mathcal{U}$.

Now, suppose that $\operatorname{FLIP}(\mathcal{U})={ }_{f} \mathcal{U}$ and $\operatorname{FLIP}(\mathcal{U})={ }_{r} \mathcal{U}$. Let $w^{*} \in \mathcal{U}$ minimize $f$ over $\mathcal{U}$. Lemma 5 and the equation $\operatorname{FLIP}(\mathcal{U})={ }_{f} \mathcal{U}$ imply that $w^{*} \in \operatorname{FLIP}(\mathcal{U}) \cap$ $\operatorname{GP}(\operatorname{FltP}(\mathcal{U}))$. Note $x, x+u^{1} \in \operatorname{intr}(\operatorname{GP}(\mathcal{U}))$ by the definition of Case 4, so neither are cut by $z$ or $z+u^{1}$. Thus, $\operatorname{GP}(\operatorname{FLIP}(\mathcal{U})) \cap \operatorname{FLIP}(\mathcal{U})$ contains a least two vectors: $w^{*}$ and one of $x$ or $x+u^{1}$. The set FLIP $(\mathcal{U})$ can only be disconnected if $\operatorname{GP}(\operatorname{FLIP}(\mathcal{U})) \cap \operatorname{FLIP}(\mathcal{U})=\left\{z, x+u^{1}\right\}$ or $\operatorname{GP}(\operatorname{FLIP}(\mathcal{U})) \cap \operatorname{FLIP}(\mathcal{U})=\left\{z+u^{1}, x\right\}$. The first setting implies that $x+u^{1}$ strictly cuts $x$ and $z+u^{1}$ but not $z$, which is not possible by Lemma 1 (iv). The second setting implies that $x$ strictly cuts $x+u^{1}$ and $z$ but not $z+u^{1}$, which is again not possible. This proves (iii).

\subsection{An analysis of Case $\mathbf{5}$.}

We begin by showing

$$
\begin{aligned}
H^{1} \cap \mathbb{Z}^{2} & =\emptyset \text { or } H^{-1} \cap \mathbb{Z}^{2}=\emptyset ; \\
\text { if } H^{1} \cap \mathbb{Z}^{2} & \neq \emptyset \text {, then }\left\{z-u^{1}+u^{2}\right\}=H^{1} \cap \mathbb{Z}^{2}, \text { and } \\
\text { if } H^{-1} \cap \mathbb{Z}^{2} & \neq \emptyset \text {, then }\left\{z+u^{1}-u^{2}\right\}=H^{-1} \cap \mathbb{Z}^{2} .
\end{aligned}
$$

The first statement in (12) shows that the two outcomes in Case 5 cannot both be satisfied. Let $x:=z+k u^{1}-u^{2} \in H^{-1} \cap \mathbb{Z}^{2}$. If $k \leq 0$, then $z \in$ $\operatorname{conv}\left\{x, z+u^{1}, z+u^{2}\right\}$ which contradicts $z \notin \operatorname{intr}(\operatorname{GP}(\mathcal{U}))$. If $k \geq 2$, then $z+u^{1} \in \operatorname{conv}\left\{x, z+u^{2}, z\right\}$ which contradicts $z+u^{1} \notin \operatorname{intr}(\operatorname{GP}(\mathcal{U}))$. Thus, $H^{-1} \cap \mathbb{Z}^{2} \subseteq\left\{z+u^{1}-u^{2}\right\}$. Similarly, $H^{1} \cap \mathbb{Z}^{2} \subseteq\left\{z-u^{1}+u^{2}\right\}$. If $\left\{z+u^{1}-u^{2}\right\}=$ $H^{-1} \cap \mathbb{Z}^{2}$ and $\left\{z-u^{1}+u^{2}\right\}=H^{1} \cap \mathbb{Z}^{2}$, then $z \in \operatorname{conv}\left\{z+u^{1}-u^{2}, z-u^{1}+u^{2}\right\} \subseteq$ $\operatorname{intr}(\mathrm{GP}(\mathcal{U}))$, which is a contradiction. This proves (12). 


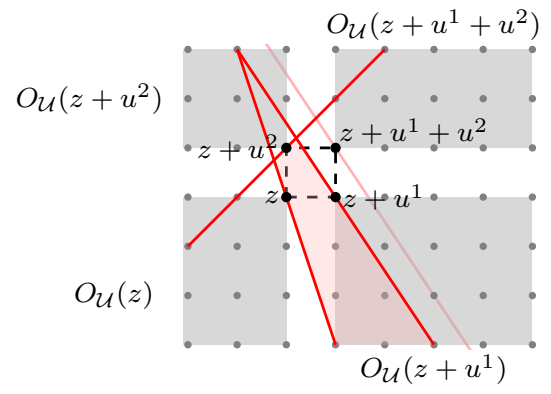

(i)

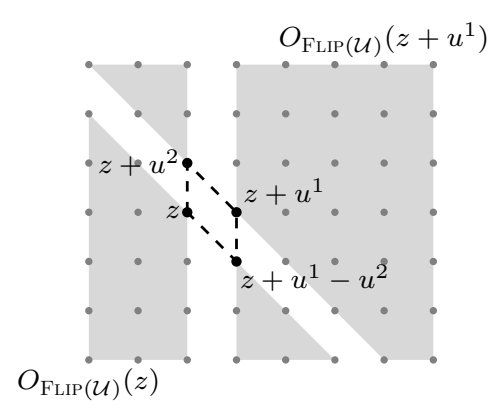

(ii)

Fig. 7 (i) The orthants used to prove Theorem 3 in Case 5 GP $(\mathcal{U})$ is also drawn. (ii) The orthants of $\operatorname{FLIP}(\mathcal{U})$ when $\left|H^{-1} \cap \operatorname{GP}(\mathcal{U})\right| \geq 1$.

\section{Lemma 13 Suppose $\mathcal{U}$ fits into Case 5. Then}

(i) $\operatorname{FLIP}(\mathcal{U}) \leq_{r} \mathcal{U}$.

(ii) if $\mathcal{U}$ does not contain a minimizer of (CM), then $\operatorname{FLIP}(\mathcal{U})<_{r} \mathcal{U}$

(iii) if $\operatorname{FLIP}(\mathcal{U})=_{f} \mathcal{U}$ and $\operatorname{FLIP}(\mathcal{U})=_{r} \mathcal{U}$, then $\operatorname{FLIP}(\mathcal{U})$ is connected and $\operatorname{FLIP}(\mathcal{U}) \cap \operatorname{GP}(\operatorname{FLIP}(\mathcal{U})) \backslash \mathcal{U} \neq \emptyset$.

Proof First, we show that $\operatorname{FLIP}(\mathcal{U}) \leq_{r} \mathcal{U}$. By Lemma 6 we assume that $\mathcal{U}$ does not contain a minimizer of (CM). By Lemma $10(i), z^{*} \in \operatorname{intr}(\operatorname{GP}(\mathcal{U}))$.

It was shown in (12) that either $\operatorname{FLIP}(U)=\left(u^{1}-u^{2}, u^{2}\right)$ or $\operatorname{FLIP}(U)=$ $\left(u^{1},-u^{1}+u^{2}\right)$, depending on if $H^{-1} \cap \mathbb{Z}^{2} \neq \emptyset$ or $H^{1} \cap \mathbb{Z}^{2} \neq \emptyset$, respectively. These outcomes are symmetric, so we assume

$$
\operatorname{FLIP}(U)=\left(u^{1}-u^{2}, u^{2}\right) \text { and } \operatorname{FLIP}(\mathcal{U})=\left\{z, z+u^{1}, z+u^{2}, z+u^{1}-u^{2}\right\} .
$$

Figure 7 illustrates this setting.

If $z^{*} \in O_{\mathrm{FuIP}(\mathcal{U})}(z)$, then $z \in \operatorname{conv}\left\{z^{*}, z+u^{1}-u^{2}, z+u^{2}\right\}$ yielding the contradiction $z \in \operatorname{intr}(\operatorname{GP}(\mathcal{U}))$. Similarly, if $z^{*} \in O_{\mathrm{FLIP}(\mathcal{U})}\left(z+u^{2}\right)$, then we obtain the contradiction $z+u^{2} \in \operatorname{intr}(\operatorname{GP}(\mathcal{U}))$ and if $z^{*} \in O_{\mathrm{FLIP}(\mathcal{U})}\left(z+u^{1}\right)$, then we obtain the contradiction $z+u^{1} \in \operatorname{intr}(\operatorname{GP}(\mathcal{U}))$. Hence, $z^{*} \in O_{\mathrm{FLIP}(\mathcal{U})}(z+$ $\left.u^{1}-u^{2}\right) \subseteq O_{\mathcal{U}}\left(z+u^{1}\right)$. We can write $z^{*}$ as

$$
\begin{aligned}
z^{*} & =\left(z+u^{1}\right)+k_{1} u^{1}+k_{2}\left(-u^{2}\right) \\
& =\left(z+u^{1}-u^{2}\right)+k_{1}\left(u^{1}-u^{2}\right)+\left(k_{2}-k_{1}-1\right)\left(-u^{2}\right),
\end{aligned}
$$

where $k_{1}, k_{2} \geq 0$ and $k_{1}+k_{2} \geq 1$. Note that $k_{2}-k_{1}-1 \geq 0$ because $z^{*} \in$ $O_{\mathrm{FLIP}(\mathcal{U})}\left(z+u^{1}-u^{2}\right)$. Hence,

$$
r_{\mathcal{U}}\left(z^{*}\right)=k_{1}+k_{2}>k_{2}-1=r_{\operatorname{FLP}(\mathcal{U})}\left(z^{*}\right) .
$$

This shows that $\operatorname{FLIP}(\mathcal{U})<_{r} \mathcal{U}$.

Now, suppose that $\operatorname{FLIP}(\mathcal{U})={ }_{f} \mathcal{U}$ and $\operatorname{FLIP}(\mathcal{U})={ }_{r} \mathcal{U}$. Let $w^{*} \in \mathcal{U}$ minimize $f$ over $\mathcal{U}$. Lemma $\left[5\right.$ and the equation $\operatorname{FLIP}(\mathcal{U})={ }_{f} \mathcal{U}$ imply that $w^{*} \in \operatorname{FLIP}(\mathcal{U}) \cap$ $\operatorname{GP}(\operatorname{Flip}(\mathcal{U}))$. Furthermore, because $z+u^{1}-u^{2} \in H^{-1} \subseteq \operatorname{intr}\left(\operatorname{GP}\left(\mathcal{U}^{i}\right)\right)$ it is not 
cut by $z, z+u^{2}$, or $z+u^{1}$. Thus, $\operatorname{FLIP}(\mathcal{U}) \cap \operatorname{GP}(\operatorname{FLIP}(\mathcal{U}))$ contains at least two vectors: $w^{*}$ and $z+u^{1}-u^{2}$. $\operatorname{FLIP}(\mathcal{U})$ can only be disconnected if $w^{*}=z+u^{2}$. However, $z+u^{2}$ does not strictly cut $z$ or $z+u^{1}$. Therefore, $z+u^{1}-u^{2}$ strictly cuts $z$ and $z+u^{1}$ but not $z+u^{2}$. This is not possible by Lemma 1 (iv). Thus, $\operatorname{FLIP}(\mathcal{U})$ is connected and $z+u^{1}-u^{2} \in \operatorname{FLIP}(\mathcal{U}) \cap \operatorname{GP}(\operatorname{FLIP}(\mathcal{U})) \backslash \mathcal{U}$.

\section{Convergence proofs}

4.1 Convergence to a minimum: The proof of Theorem 1

Recall that if $\mathcal{U}$ contains a vector $v$ with $\nabla f(v)=\mathbf{0}$, then we do not update $\mathcal{U}$. The fact that each step uses constantly many gradient evaluations follows directly from counting the number of gradient evaluations in every case in Table 1 Cases 3 to 5 require the most evaluations: 12 to determine $\mathcal{U} \cap \operatorname{GP}(\mathcal{U})$ and 8 to determine $H^{-1}$ and $H^{1}$. If $\mathcal{U}$ does not fit into the table, then it is lattice-free by Lemma 4 . Using the notation $\leq_{f}$ and $\leq_{r}$, we restate Theorem 1 (i) and (ii) as Theorem 3. The proof of Theorem 3 follows directly from Lemmata 7, 9, 11, 12, and 13.

Theorem 3 Let $\mathcal{U}$ be preprocessed as in Lemma 2. If $\mathcal{U}$ fits into Table 1 and does not contain an optimal solution of (CM), then at least one of the following holds:

(i) $\operatorname{FLIP}(\mathcal{U})<_{f} \mathcal{U}$ and $\operatorname{FLIP}(\mathcal{U}) \leq_{r} \mathcal{U}$

(ii) $\operatorname{FLIP}(\mathcal{U}) \leq_{f} \mathcal{U}$ and $\operatorname{FLIP}(\mathcal{U})<_{r} \mathcal{U}$

(iii) $\operatorname{FLIP}(\mathcal{U}) \leq_{f} \mathcal{U}, \operatorname{FLIP}(\mathcal{U}) \leq_{r} \mathcal{U}$, and $\operatorname{FLIP}(\mathcal{U})$ is connected.

If $\mathcal{U}$ is connected, then $\operatorname{FLIP}(\mathcal{U})$ satisfies (ii).

Theorem 3 states that either $\leq_{f}$ or $\leq_{r}$ decreases after at most two updates. Assumption (11) implies that $\leq_{f}$ and $\leq_{r}$ can only decrease a finite number of times before $\mathcal{U}$ contains a minimizer of (CM).

Theorem 4 If $\mathcal{U}^{0}$ is unimodular and $\left(\mathcal{U}^{i}\right)_{i=0}^{\infty}$ is constructed using Table 1 , then there exists an index $T_{1}$ such that $\mathcal{U}^{i}$ contains an optimal solution of (CM) for all $i \geq T_{1}$.

We end this section with a convergence result. Suppose $f$ is $L$-Lipschitz continuous and $c$-strongly convex. We say $f$ is $c$-strongly convex for $c>0$ if

$$
f(\bar{z}) \geq f(z)+\nabla f(z)^{\top}(\bar{z}-z)+c \cdot\|\bar{z}-z\|_{2}^{2} \quad \forall z, \bar{z} \in \mathbb{R}^{2} .
$$

Denote the identity matrix by $\mathbb{I}^{2}$.

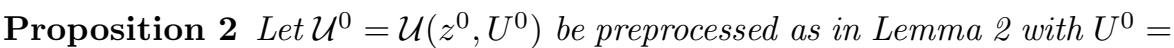
$\mathbb{I}^{2}$. Let $z^{*} \in \mathbb{Z}^{2}$ be an optimal solution to (CM). After $T_{1} \leq(6 L / c+2) \cdot r_{\mathcal{U}^{0}}\left(z^{*}\right)$ many updates via Table 1, the set $\mathcal{U}^{T_{1}}$ contains an optimal solution to (CM). The set $\mathcal{U}^{T_{1}}$ is not necessarily lattice-free. 
Proof Let $i \geq 0$ and consider updating $\mathcal{U}^{i}=\left(z^{i}, U^{i}\right)$ to $\operatorname{FLIP}\left(\mathcal{U}^{i}\right)=\mathcal{U}^{i+1}$. If $\mathcal{U}^{i}$ is connected, which occurs in Cases 3 to 5 , then $\mathcal{U}^{i+1}<_{r} \mathcal{U}^{i}$ by Lemmata 11, 12, and 13.

Analysis of the disconnected cases uses the following argument. If $z, w \in \mathbb{Z}^{2}$ are such that $z$ strictly cuts $w$, then by strong convexity we have

$$
f(w) \geq f(z)+\nabla f(z)^{\top}(w-z)+c \cdot\|w-z\|_{2}^{2}>f(z)+c .
$$

Hence, the difference between $f(w)$ and $f(z)$ is at least $c$.

Case 2 has three outcomes. If the third outcome occurs, i.e., $\operatorname{FLIP}(U)=$ $\left(-u^{1}, 2 u^{1}+u^{2}\right)$, then $\mathcal{U}^{i+1}<_{r} \mathcal{U}^{i}$ by Lemma 9. Otherwise, Lemma 10 states that $\mathcal{U}^{i+1}$ is connected, in which case $\mathcal{U}^{i+2}<_{r} \mathcal{U}^{i+1} \leq_{r} \mathcal{U}^{i}$, or the vector $w^{*}$ minimizing $f$ over $\mathcal{U}^{i}$ is strictly cut by some vector in $\mathcal{U}^{i+1}$, in which case

$$
\min \left\{f(w): w \in \mathcal{U}^{i+1}\right\}+c \leq \min \left\{f(w): w \in \mathcal{U}^{i}\right\} .
$$

Consider Case 1, By Lemma 8, either $\mathcal{U}^{i+1}$ is connected, in which case $\mathcal{U}^{i+2}<_{r} \mathcal{U}^{i+1} \leq_{r} \mathcal{U}^{i}$, or the vector $w^{*}$ minimizing $f$ over $\mathcal{U}^{i}$ is strictly cut by some vector in $\mathcal{U}^{i+1}$, in which case (13) holds.

Overall we have shown that either $\mathcal{U}^{i+2}<_{r} \mathcal{U}^{i}$ or (13) holds, independent of the case $\mathcal{U}^{i}$ falls into. The value with respect to $\leq_{r}$ can decrease by at most $r_{\mathcal{U}^{0}}\left(z^{*}\right)$. The minimum function value can decrease by at most the amount $\delta^{f}-f\left(z^{*}\right)$, where $w^{*} \in \mathcal{U}^{0}$ satisfies

$$
\delta^{f}:=f\left(w^{*}\right)=\min \left\{f(w): w \in \mathcal{U}^{0}\right\} .
$$

Suppose $v^{*} \in \mathcal{U}^{0}$ satisfies $\left\|v^{*}-z^{*}\right\|_{1}=r_{\mathcal{U}^{0}}\left(z^{*}\right)$. Because $U^{0}=\mathbb{I}^{2}$ we have $\left\|w^{*}-v^{*}\right\|_{1} \leq 2$ and

$\delta^{f}-f\left(z^{*}\right)=f\left(w^{*}\right)-f\left(z^{*}\right) \leq L \cdot\left\|w^{*}-z^{*}\right\|_{2} \leq L \cdot\left\|w^{*}-z^{*}\right\|_{1} \leq L \cdot\left(r_{\mathcal{U}^{0}}\left(z^{*}\right)+2\right)$.

If $r_{\mathcal{U}^{0}}\left(z^{*}\right)=0$, then the lemma is trivially true. If $r_{\mathcal{U}^{0}}\left(z^{*}\right) \geq 1$, then $\mathcal{U}^{i}$ must contain a minimizer of (CM) after at most

$2\left[\frac{\delta^{f}-f\left(z^{*}\right)}{c}+r_{\mathcal{U}^{0}}\left(z^{*}\right)\right] \leq 2\left[\frac{L \cdot\left(r_{\mathcal{U}^{0}}\left(z^{*}\right)+2\right)}{c}+r_{\mathcal{U}^{0}}\left(z^{*}\right)\right] \leq\left(\frac{6 L}{c}+2\right) r_{\mathcal{U}^{0}}\left(z^{*}\right)$

many updates.

4.2 Convergence towards a lattice-free set: The proof of Theorem 2 .

Let $\mathcal{U}^{0}$ be preprocessed as in Lemma 2. For $i \geq 0$ set $\mathcal{U}^{i+1}=\operatorname{FLIP}\left(\mathcal{U}^{i}\right)$ and preprocess $\mathcal{U}^{i+1}$ via Lemma 2, By Theorem 4 and Lemma 5, there exists $T_{1} \geq 1$ such that $\mathcal{U}^{i} \cap \operatorname{GP}\left(\mathcal{U}^{i}\right)$ contains an optimal solution of (CM) for all $i \geq T_{1}$. Relabeling indices allows us to assume $T_{1}=0$. After at most one flip each $\mathcal{U}^{i}$ is also connected.

Lemma $14 \operatorname{FLIP}\left(\mathcal{U}^{i}\right)$ is connected for all $i \geq 1$. 
Proof By Lemma 5, $\mathcal{U}^{i}$ contains a minimizer of (CM) for all $i \geq 0$. Hence, $\mathcal{U}^{i}={ }_{f} \mathcal{U}^{i+1}=0$ and $\mathcal{U}^{i}={ }_{f} \mathcal{U}^{i+1}=0$. Connectivity of $\mathcal{U}^{i+1}$ then follows from Lemmata 7, 9, 11, 12, and 13,

We finish with the proof of Theorem 2 .

Proof (of Theorem 2) By Theorem 4 and Lemma 14, we may assume that $\mathcal{U}^{i}$ contains a minimizer $z^{*}$ of (CM) and that $\mathcal{U}^{i}$ is connected for each $i \geq 0$. For each $i \geq 0$ define the sets

$\mathcal{M}_{1}^{i}:=\left\{w \in \mathcal{U}^{i}: f(w)=f\left(z^{*}\right)\right\}$, and

$\mathcal{M}_{j}^{i}:=\left\{w \in \mathcal{U}^{i}: w\right.$ minimizes $f$ over $\left.\mathcal{U}^{i} \cap \operatorname{GP}\left(\mathcal{U}^{i}\right) \backslash \cup_{k=1}^{j-1} \mathcal{M}_{j}^{i}\right\} \forall j \in\{2,3,4\}$.

By Lemma $5 z^{*} \in \mathcal{M}_{1}^{i} \subseteq \mathcal{M}_{1}^{i+1}$. There exists some $i$ such that $\mathcal{M}_{1}^{i}=\mathcal{M}_{1}^{i+1}$ for all $i \geq 1$. After reindexing, we assume $\mathcal{M}_{1}^{i}=\mathcal{M}_{1}^{i+1} \neq \emptyset$ for all $i \geq 1$.

Every time we update $\mathcal{U}^{i}$ via Table1, it fits into Cases 3 to 5 . Parts (iii) in Lemmata 11, 12 and 13 imply that there exists $w \in \mathcal{U}^{i+1} \cap\left(\operatorname{GP}\left(\operatorname{FLIP}\left(\mathcal{U}^{i+1}\right)\right) \backslash\right.$ $\left.\mathcal{U}^{i}\right)$. Note $w \notin \mathcal{M}_{1}^{i+1}$, otherwise $\mathcal{M}_{1}^{0} \subsetneq \mathcal{M}_{1}^{i+1}$, and $w$ does not strictly cut any vector in $\mathcal{M}_{1}^{i}$ by Proposition 1. Assumption (1) then implies that there exists some $i \geq 1$ such that the function value defining $\mathcal{M}_{2}^{i}$ is minimized. Furthermore, there exists an index $i^{*} \geq 1$ such that $\mathcal{M}_{2}^{i}=\mathcal{M}_{2}^{i+1}$ for all $i \geq i^{*}$. After reindexing, we may assume $\mathcal{M}_{2}^{i}=\mathcal{M}_{2}^{i+1} \neq \emptyset$ for all $i \geq 0$.

If $\mathcal{U}^{0}$ still fits into the table, then we may repeat the previous process to assume $\mathcal{M}_{3}^{i}=\mathcal{M}_{3}^{i+1} \neq \emptyset$ for all $i \geq 0$. Again, if $\mathcal{U}^{0}$ fits into the table, then we may repeat this one last time to assume $\mathcal{M}_{4}^{i}=\mathcal{M}_{4}^{i+1} \neq \emptyset$ for all $i \geq 0$. Recall that we do not update $\mathcal{U}^{i}$ if it contains a vector $v$ with $\nabla f(v)=\overline{\mathbf{0}}$ or it does not fit into the table, and in both situations $\operatorname{GP}\left(\mathcal{U}^{i}\right)$ is lattice-free by Lemma 4. If we reach the step when $\mathcal{M}_{4}^{i}=\mathcal{M}_{4}^{i+1} \neq \emptyset$ for all $i \geq 0$, then the disjoint sets $\mathcal{M}_{1}^{0}, \mathcal{M}_{2}^{0}, \mathcal{M}_{0}^{3}$ and $\mathcal{M}_{0}^{4}$ are all non-empty. These four sets are contained in $\mathcal{U}^{0}$. Hence, $\operatorname{GP}\left(\mathcal{U}^{0}\right)$ is lattice-free by Corollary 2,

\section{Conclusions}

The results in this paper provide the first method for updating gradient polyhedra in dimension two. The results extend to non-differentiable functions using subgradients. Theorem 3 tells us that if we 'flip' as defined in Table 1 until we no longer satisfy a case in the table, then we have an optimality certificate for (CM) in the form of a lattice-free gradient polyhedron. This procedure mimics gradient descent in the following ways: each update is represented as a gradient polyhedron with at most $2^{n}$ facets which meets the bounds given by the theory, each update only needs constantly many gradient evaluations, and the initial set can be an arbitrary unimodular set. There are many open questions. For instance, it may be possible to adjust the 'step size' of each flip to achieve faster convergence. We believe this can be analyzed whenever $\mathcal{U}$ is such that multiple successive flips $\operatorname{FLIP}(\mathcal{U}), \operatorname{FLIP}(\operatorname{FLIP}(\mathcal{U})), \ldots$ fall into the same case. Other questions include if the measure $\leq_{r}$ or the updates can be extended to $n \geq 3$ or to models with additional constraints. 


\section{References}

1. Andersen, K., Louveaux, Q., Weismantel, R., Wolsey, L.A.: Inequalities from two rows of a simplex tableau. In: Fischetti M., Williamson D.P. (eds.) Integer Programming and Combinatorial Optimization (IPCO). Lecture Notes in Computer Science, vol. 4513. Springer, Berlin, Heidelberg (2007)

2. Averkov, G., Krümpelmann, J., Weltge, S.: Notions of maximality for integral latticefree polyhedra: the case of dimension three. Mathematics of Operations Research pp. 1035-1062 (2017)

3. Averkov, G., Wagner, C., Weismantel, R.: Maximal lattice-free polyhedra: Finiteness and an explicit description in dimension three. Mathematics of Operations Research pp. 721-742 (2011)

4. Baes, M., Oertel, T., Wagner, C., Weismantel, R.: Mirror-descent methods in mixedinteger convex optimization. In: Jünger M., Reinelt G. (eds) Facets of Combinatorial Optimization, pp. 101-131. Springer, Berlin, Heidelberg (2013)

5. Baes, M., Oertel, T., Weismantel, R.: Duality for mixed-integer convex minimization. Mathematical Programming Series A 158, 547 - 564 (2016)

6. Balas, E.: Intersection cuts - a new type of cutting planes for integer programming. Operations Research 19(1), 19 - 39 (1971)

7. Barvinok, A.: A Course in Convexity, vol. 54. Graduate Studies in Mathematics, American Mathematical Society, Providence, Rhode Island (2002)

8. Basu, A., Conforti, M., Cornuéjols, G., Weismantel, R., Weltge, S.: Optimality certificates for convex minimization and Helly numbers. Operations Research Letters 45, 671-674 (2017)

9. Basu, A., Conforti, M., Cornuéjols, G., Zambelli, G.: Maximal lattice-free convex sets in linear subspaces. Mathematics of Operations Research 35(3), 704-720 (2010)

10. Basu, A., Conforti, M., Di Summa, M.: A geometric approach to cut-generating functions. Mathematical Programming 151(1), 153 - 189 (2015)

11. Basu, A., Cornuéjols, G., Köppe, M.: Unique minimal liftings for simplicial polytopes. Mathematics of Operations Research 37(2), 346-355 (2012)

12. Bell, D.: A theorem concerning the integer lattice. Studies in Applied Mathematics 56 , 187-188 (1977)

13. Bonami, P., Biegler, L.T., Conn, A.R., Cornuéjols, G., Grossman, I.E., Laird, C.D., Lee, J., Lodi, A., Margot, F., Sawaya, N., Wächter, A.: An algorithmic framework for convex mixed integer nonlinear programs. Discrete Optimization 5(2), 186-204 (2008)

14. Boyd, S., Vandenberghe, L.: Convex Optimization. Cambridge University Press (2004)

15. Del Pia, A., Weismantel, R.: Relaxations of mixed integer sets from lattice-free polyhedra. 4OR 10(3), 221-244 (2012)

16. Dey, S.S., Wolsey, L.A.: Lifting integer variables in minimal inequalities corresponding to lattice-free triangles. In: Lodi A., Panconesi A., Rinaldi G. (eds.) International Conference on Integer Programming and Combinatorial Optimization (IPCO). Lecture Notes in Computer Science, vol. 5035, pp. 463 - 475. Springer, Berlin, Heidelberg (2008)

17. Doignon, J.: Convexity in cristallographical lattices. Journal of Geometry 3, 71-85 (1973)

18. Duran, M.A., Grossman, I.E.: An outer-approximation algorithm for a class of mixedinteger nonlinear programs. Mathematical Programming 36(3), 307-339 (1986)

19. Grötschel, M., Lovász, L., Schrijver, A.: Geometric Algorithms and Combinatorial Optimization, Algorithms and Combinatorics, vol. 2. Springer-Verlag Berlin Heidelberg, 1 edn. (1988)

20. Gupta, O., Ravindran, V.: Branch and bound experiments in convex nonlinear integer programming. Management Science 31, 1533-1546 (1985)

21. Leyffer, S.: Integrating SQP and branch-and-bound for mixed integer nonlinear programming. Computational Optimization and Applications 18, 295-309 (2001)

22. Lovász, L.: Geometry of numbers and integer programming. In: M.Iri, Tanabe, K. (eds.) Mathematical Programming: Recent Developments and Applications. pp. $177-201$. Kluwer Academic Publishers (1989)

23. Scarf, H.: An observation on the structure of production sets with indivisibilities. Proceedings of the National Academy of Sciences of the United States of America 74, 3637-3641 (1977) 